\title{
First broadband characterization and redshift determination of the VHE blazar MAGIC J2001+439 *
}

J. Aleksić ${ }^{1}$, S. Ansoldi ${ }^{2}$, L. A. Antonelli ${ }^{3}$, P. Antoranz ${ }^{4}$, A. Babic ${ }^{5}$, P. Bangale ${ }^{6}$, U. Barres de Almeida ${ }^{6}$, J. A. Barrio ${ }^{7}$, J. Becerra González $^{8,25, \star \star}$, W. Bednarek ${ }^{9}$, E. Bernardini1 ${ }^{10}$, A. Biland ${ }^{11}$, O. Blanch ${ }^{1}$, S. Bonnefoy ${ }^{7}$, G. Bonnoli ${ }^{3}$, F. Borracci ${ }^{6}$, T. Bretz ${ }^{12,26}$, E. Carmona $^{13}$, A. Carosi $^{3}$,

D. Carreto Fidalgo ${ }^{7}$, P. Colin ${ }^{6}$, E. Colombo ${ }^{8}$, J. L. Contreras ${ }^{7}$, J. Cortina ${ }^{1}$, S. Covino ${ }^{3}$, P. Da Vela ${ }^{4}$, F. Dazzi ${ }^{6}$, A. De Angelis ${ }^{2}$, G. De Caneva ${ }^{10}$,

B. De Lotto ${ }^{2}$, C. Delgado Mendez ${ }^{13}$, M. Doert ${ }^{14}$, A. Domínguez ${ }^{15,27}$, D. Dominis Prester ${ }^{5}$, D. Dorner ${ }^{12}$, M. Doro ${ }^{16}$, S. Einecke $^{14}, D^{2}$ Eisenacher $^{12}$, D. Elsaesser ${ }^{12}$, E. Farina ${ }^{17}$, D. Ferenc ${ }^{5}$, M. V. Fonseca ${ }^{7}$, L. Font $^{18}$, K. Frantzen ${ }^{14}$, C. Fruck ${ }^{6}$, R. J. García López ${ }^{8}$, M. Garczarczyk ${ }^{10}$, D. Garrido Terrats $^{18}$, M. Gaug ${ }^{18}$, N. Godinović ${ }^{5}$, A. González Muñoz ${ }^{1}$, S. R. Gozzini ${ }^{10}$, D. Hadasch ${ }^{19}$, M. Hayashida ${ }^{20}$, J. Herrera ${ }^{8}$, A. Herrero ${ }^{8}$, D. Hildebrand ${ }^{11}$, J. Hose ${ }^{6}$, D. Hrupec ${ }^{5}$, W. Idec ${ }^{9}$, V. Kadenius ${ }^{21}$, H. Kellermann ${ }^{6}$, K. Kodani2 ${ }^{20 \star \star}$, Y. Konno ${ }^{20}$, J. Krause ${ }^{6}$, H. Kubo $^{20}$,

J. Kushida ${ }^{20}$, A. La Barbera ${ }^{3}$, D. Lelas ${ }^{5}$, N. Lewandowska ${ }^{12}$, E. Lindfors ${ }^{21,28}$, S. Lombardi ${ }^{3}$, M. López ${ }^{7}$, R. López-Coto ${ }^{1}$, A. López-Oramas ${ }^{1}$, E. Lorenz ${ }^{6}$, I. Lozano ${ }^{7}$, M. Makariev ${ }^{22}$, K. Mallot ${ }^{10}$, G. Maneva ${ }^{22}$, N. Mankuzhiyil ${ }^{2}$, K. Mannheim ${ }^{12}$, L. Maraschi ${ }^{3}$, B. Marcote ${ }^{23}$, M. Mariotti ${ }^{16}$, M. Martínez ${ }^{1}$, D. Mazin ${ }^{6}$, U. Menzel ${ }^{6}$, M. Meucci ${ }^{4}$, J. M. Miranda ${ }^{4}$, R. Mirzoyan ${ }^{6}$, A. Moralejo ${ }^{1}$, P. Munar-Adrover ${ }^{23}$, D. Nakajima ${ }^{20}$,

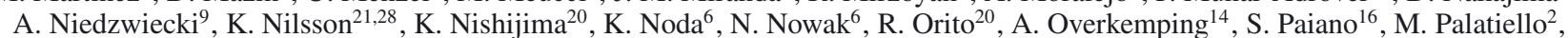
D. Paneque ${ }^{6, \star \star}$, R. Paoletti ${ }^{4}$, J. M. Paredes ${ }^{23}$, X. Paredes-Fortuny ${ }^{23}$, S. Partini ${ }^{4}$, M. Persic ${ }^{2,29}$, F. Prada ${ }^{15,30}$, P. G. Prada Moroni ${ }^{24}$, E. Prandini ${ }^{11}$, S. Preziuso ${ }^{4}$, I. Puljak ${ }^{5}$, R. Reinthal ${ }^{21}$, W. Rhode ${ }^{14}$, M. Ribó ${ }^{23}$, J. Rico ${ }^{1}$, J. Rodriguez Garcia ${ }^{6}$, S. Rügamer ${ }^{12}$, A. Saggion ${ }^{16}$, T. Saito $^{20}$, K. Saito $^{20}$, K. Satalecka ${ }^{7}$, V. Scalzotto ${ }^{16}$, V. Scapin ${ }^{7}$, C. Schultz ${ }^{16}$, T. Schweizer ${ }^{6}$, S. N. Shore ${ }^{24}$, A. Sillanpää ${ }^{21}$, J. Sitarek ${ }^{1}$, I. Snidaric ${ }^{5}$, D. Sobczynska ${ }^{9}$, F. Spanier ${ }^{12}$, V. Stamatescu ${ }^{1}$, A. Stamerra ${ }^{3}$, T. Steinbring ${ }^{12}$, J. Storz ${ }^{12}$, M. Strzys ${ }^{6}$, S. Sun ${ }^{6}$, T. Surić ${ }^{5}$, L. Takalo ${ }^{21}$, H. Takami $^{20}$, F. Tavecchio ${ }^{3}$,

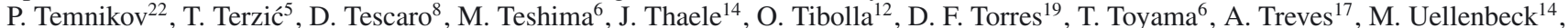
P. Vogler ${ }^{11}$, R. M. Wagner ${ }^{6,31}$, F. Zandanel ${ }^{15,32}$, R. Zanin ${ }^{23}$ (The MAGIC Collaboration), F. D’ Ammando ${ }^{33,34}$, T. Hovatta ${ }^{35}$, V. M. Larionov ${ }^{36,37,38}$, W. Max-Moerbeck ${ }^{39}$, M. Perri ${ }^{3,40}$, A. C. S. Readhead ${ }^{35}$, J. L. Richards ${ }^{41}$, T. Sakamoto ${ }^{42}$, R. D. Schwartz ${ }^{43 \dagger}$, F. Verrecchia ${ }^{40}$, and L. C. Reyes ${ }^{44}$

(Affiliations can be found after the references)

Received 22 May 2014 / Accepted 1 September 2014

\section{ABSTRACT}

Aims. We aim to characterize the broadband emission from 2FGL J2001.1+4352, which has been associated with the unknown-redshift blazar MG4 J200112+4352. Based on its gamma-ray spectral properties, it was identified as a potential very high energy (VHE; $E>100 \mathrm{GeV}$ ) gamma-ray emitter. We investigate whether this object is a VHE emitter, characterize its gamma-ray spectrum, and study the broadband emission within the one-zone synchrotron self-Compton (SSC) scenario, which is commonly used to describe the emission in blazars. Moreover, we also intend to determine the redshift of this object, which is a crucial parameter for its scientific interpretation.

Methods. The source was observed with MAGIC first in 2009 and later in 2010 within a multi-instrument observation campaign. The MAGIC observations yielded $14.8 \mathrm{~h}$ of good quality stereoscopic data. Besides MAGIC, the campaign involved, observations with FermiLAT, Swift-XRT/UVOT, the optical telescopes KVA, Goddard Robotic Telescope, Galaxy View observatory, Crimean Astrophysical observatory, St. Petersburg observatory, and the Owens Valley Radio Observatory. The object was monitored at radio, optical and gamma-ray energies during the years 2010 and 2011. We characterize the radio to VHE spectral energy distribution and quantify the multiband variability and correlations over short (few days) and long (many months) timescales. We also organized deep imaging optical observations with the Nordic Optical Telescope in 2013 to determine the source redshift.

Results. The source, named MAGIC J2001+439, is detected for the first time at VHE with MAGIC at a statistical significance of $6.3 \sigma$ $(E>70 \mathrm{GeV})$ during a $1.3 \mathrm{~h}$ long observation on 2010 July 16 . The multi-instrument observations show variability in all energy bands with the highest amplitude of variability in the X-ray and VHE bands. Besides the variability on few-day timescales, the long-term monitoring of MAGIC $\mathrm{J} 2001+439$ shows that, the gamma-ray, optical, and radio emissions gradually decreased on few-month timescales from 2010 through 2011 , indicating that at least some of the radio, optical and gamma-ray emission is produced in a single region by the same population of particles. We also determine for the first time the redshift of this BL Lac object through the measurement of its host galaxy during low blazar activity. Using the observational evidence that the luminosities of BL Lac host galaxies are confined to a relatively narrow range, we obtain $z=0.18 \pm 0.04$. Additionally, we use the Fermi-LAT and MAGIC gamma-ray spectra to provide an independent redshift estimation, $z=0.17 \pm 0.10$. Using the former (more accurate) redshift value, we adequately describe the broadband emission with a one-zone SSC model for different activity states and interpret the few-day timescale variability as produced by changes in the high-energy component of the electron energy distribution.

Key words. galaxies: active - BL Lacertae objects: individual: MAGIC J2001+435 - BL Lacertae objects: individual: MAGIC J2001+439 gamma rays: galaxies

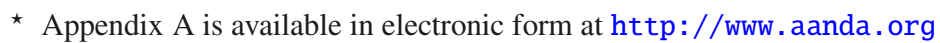

$\star \star$ Corresponding authors: K. Kodani, e-mail: kodanik.z@gmail.com; D. Paneque, e-mail: dpaneque@mppmu.mpg.de;

J. Becerra González, e-mail: jbecerragonzalez@gmail.com

$\dagger$ Deceased.
} 


\section{Introduction}

Blazars are radio-loud active galactic nuclei (AGN) with relativistic jets pointing towards the observer (see e.g. Urry \& Padovani 1995). They are the most common extragalactic sources detected in the very high energy (VHE; $E>100 \mathrm{GeV}$ ) gamma-ray range. The spectral energy distributions (SEDs) of blazars show a double-bump shape. The first bump peaks at optical/X-ray frequencies and is attributed to synchrotron radiation from relativistic electrons. On the other hand, the origin of the second bump, which peaks at gamma-ray energies, is still under debate. Leptonic models are generally favored. In these models, the high-energy (HE; $E>100 \mathrm{MeV}$ ) radiation is produced by inverse Compton (IC) of primary HE electrons scattering off low-energy photons. The origin of target lowenergy photons may be synchrotron radiation of the primary electrons themselves in the synchrotron self-Compton (SSC) scenario (Band \& Grindlay 1985; Maraschi et al. 1992; Bloom \& Marscher 1996), or seed photons produced outside of the jet in the external Compton (EC) scenario (Dermer \& Schlickeiser 1993; Sikora et al. 1994). However, hadron-driven emission is also possible (e.g. Mannheim \& Biermann 1992; Mücke et al. 2003). The emission would include proton, muon, and pion synchrotron radiation, as well as production of gamma rays from neutral pion decays, electrons, and positrons generated in charged pion decays that result from photon-hadron collisions. The hadronic models require total jet powers that are typically about 1-2 orders of magnitude higher than for the leptonic models.

Blazars are separated into two categories by the equivalent widths of their optical emission lines, BL Lac objects and flatspectrum radio quasars (FSRQs). The BL Lac objects show featureless optical spectra with weak or no emission lines that are possibly masked by a strong non-thermal emission from the relativistic jet, while the FSRQs display prominent broad emission lines in their optical spectra. The absence of emission/absorption lines makes it very difficult to determine the redshift for distant BL Lac objects, which often precludes detailed studies on the nature, intrinsic characteristics of individual objects, and substantially hampers and/or biases blazar population/unification studies.

The flux of the VHE gamma-ray photons coming from a distant source is attenuated by electron-positron pair creation due to interaction with the extragalactic background light (EBL; Gould \& Schréder 1966; Stecker 1969; Fazio \& Stecker 1970; Hauser $\&$ Dwek 2001). The EBL is the sum of the stellar and dust emission integrated over cosmic time. The EBL photon density carries information about the cosmic history of the star formation rate on galaxy evolution. Several EBL models have been proposed in the past few years (Stecker et al. 2006; Franceschini et al. 2008; Gilmore et al. 2009; Kneiske \& Dole 2010; Finke et al. 2010; Domínguez et al. 2011). The VHE gamma-ray absorption is energy dependent and increases strongly with redshift. Therefore, the observed VHE spectra from distant sources are distorted with respect to the intrinsic source spectra. The distances of unknown redshift BL Lac objects can be estimated by comparing the $\mathrm{GeV}$ and $\mathrm{TeV}$ spectra and assuming a specific EBL model (Prandini et al. 2011). This estimation is based on the measurement of the intrinsic source spectrum with the Fermi Large Area Telescope (LAT) at energies below $\sim 10-30 \mathrm{GeV}$, where there is little or no EBL absorption.

There are only $\sim 50$ blazars significantly detected at $\mathrm{VHE}^{1}$. This very low number of known VHE blazars is a consequence

\footnotetext{
http://tevcat.uchicago.edu
}

of the difficulty of performing sensitive scans over large portions of the sky with Imaging Atmospheric Cherenkov Telescopes (IACTs), which have narrow field of view $\left(3^{\circ}-5^{\circ}\right)$ cameras and only $\sim 1000 \mathrm{~h}$ of moonless time per year with good weather conditions. On the other hand, more than $1000 \mathrm{HE}$ gamma-ray emitting blazars have been detected with Fermi-LAT (Abdo et al. 2009; Nolan et al. 2012), and many of them have been identified (based on their spectral properties) as potential VHE emitting sources (Ackermann et al. 2013).

The object OFGL J2001.0+4352 was initially one of the unidentified Fermi-LAT sources included in the Fermi bright source list (Abdo et al. 2009). This source was first detected only above $1 \mathrm{GeV}$ with a photon flux $F_{1 \mathrm{GeV}}=(7.8 \pm$ 1.2) $\times 10^{-9} \mathrm{ph} \mathrm{cm}^{-2} \mathrm{~s}^{-1}$ between 1 and $100 \mathrm{GeV}$. Early on, this source was identified by the Fermi-LAT collaboration as a source expected to exhibit VHE emission, which is information that was shared with the H.E.S.S., MAGIC and VERITAS collaborations in 2009 October. This information triggered observations with MAGIC, which led to the first VHE detection of this source in 2010 July (see Mariotti et al. 2010; Berger et al. 2010, 2011). This source was initially designated MAGIC J2001+435, although we change its name to MAGIC J2001+439 in this paper to properly follow the IAU guidelines for naming astronomical objects.

The latest Fermi-LAT catalogs confirmed the brightness and hardness of the gamma-ray spectra of this source. In the second Fermi-LAT source catalog (2FGL, Nolan et al. 2012), this source is denoted 2FGL J2001.1+4352, and its spectrum is characterized with a power-law function $\mathrm{d} N / \mathrm{d} E \propto E^{-\Gamma}$ with $\Gamma=1.90 \pm 0.03$ above $100 \mathrm{MeV}$. This source is also present in the first Fermi HE LAT catalog (1FHL, Ackermann et al. 2013), where it is denoted 1FHL J2001.1+4353, and the spectrum is characterized by a power-law function with $\Gamma=2.38 \pm 0.18$ above $10 \mathrm{GeV}$, extending to VHE with a flux above $100 \mathrm{GeV}$ of $F_{100 \mathrm{GeV}}=\left(2.2_{-1.2}^{+1.9}\right) \times 10^{-11} \mathrm{ph} \mathrm{cm}^{-2} \mathrm{~s}^{-1}$ (Ackermann et al. 2013). Bassani et al. (2009) found a counterpart consistent with the radio bright source MG4 J200112 $+4352^{2}$ from the NRAO VLA Sky Survey (NVSS; Condon et al. 1998) $20 \mathrm{~cm}$ wavelength image. The object MG4 J200112+4352 is only 0.01 deg away from the location of 2FGL J2001.1+4352 (which has a 95\% confidence level position uncertainty of $0.02 \mathrm{deg}$ ) and is consistent with the Swift and the XMM Slew positions in the X-ray band. The source was identified as a BL Lac object using spectroscopic observations with the $1.52 \mathrm{~m}$ optical telescope from the Bologna Astronomical observatory and was classified as a high-frequency-peaked BL Lac object (HBL) by Bassani et al. (2009). The source redshift remained undetermined due to the low signal-to-noise ratio of their observations. Yet, they found indications of a slope change in the optical spectrum, which could be interpreted as a non-thermal component merging with the light from the host galaxy. Based on this feature, they gave a rough estimate of $\sim 0.2$ for the redshift of this source. More recently, Shaw et al. (2013) used higher quality optical observations to derive a lower limit for the redshift of $z>0.11$, based on the non-detection of the host galaxy, which was assumed to be a giant elliptical galaxy with an absolute $R$-band magnitude of $M_{R}=-22.5 \pm 0.5$.

In this paper, we report the results from a multiwavelength (MWL) campaign from summer 2010, providing coverage from the radio up to the VHE gamma-ray band and

2 MG4 J200112+4352 is located at RA(J2000) $=20 \mathrm{~h} 01 \mathrm{~m} 12.9 \mathrm{~s}$, $\operatorname{Dec}(\mathrm{J} 2000)=+43 \mathrm{~d} 52 \mathrm{~m} 53 \mathrm{~s}$ (NASA/IPAC Extragalactic Database, http://ned.ipac.caltech.edu). 

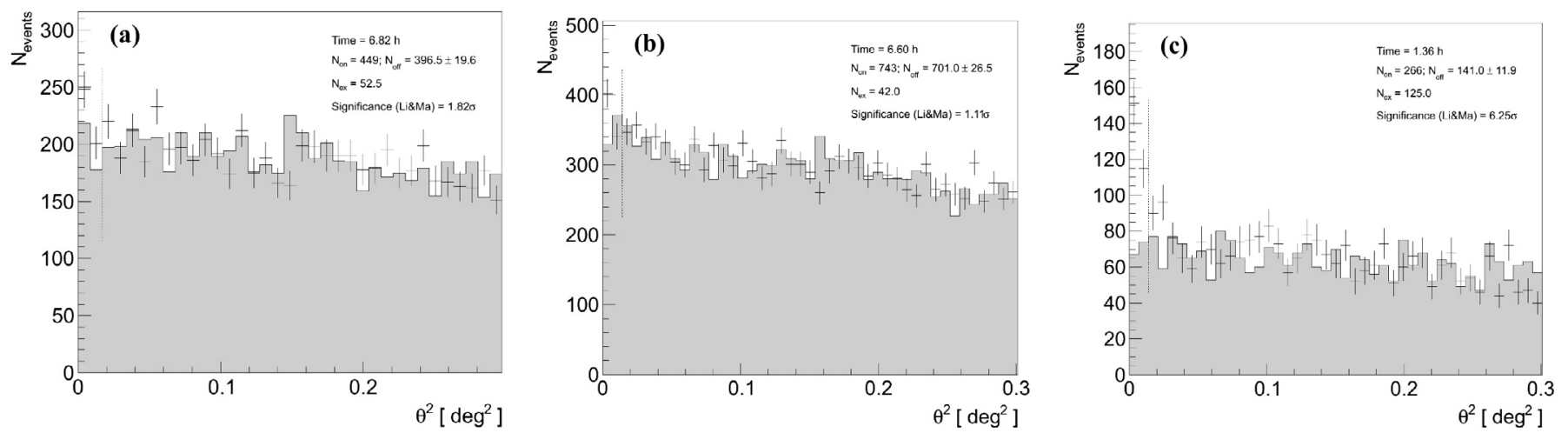

Fig. 1. Theta-squared distributions of MAGIC J2001+439 observed on 2009 November (panel a), energy threshold is $E>100 \mathrm{GeV}$ ) between July and September excluding 2010 July 16 (panel b), $E>70 \mathrm{GeV}$ ) and on $2010 \mathrm{July} 16$ (panel c), $E>70 \mathrm{GeV}$ ). Crosses represent the event distribution from the source, while the gray histogram the measured background. The signal region is indicated by the vertical dotted line.

leading to the first VHE detection of this source. The multiinstrument observations allowed us to characterize, the radio to VHE broadband SED of this object for the first time. We also report on the multiband variability and correlation properties during this campaign and follow-up observations performed during the years 2010 and 2011. Moreover, we report the first measurement of the redshift for this source through the detection of its host galaxy with the $2.5 \mathrm{~m}$ Nordic Optical Telescope (NOT) during low blazar activity. Additionally, we also estimate the redshift of this object using the HE and VHE gamma-ray spectra, as measured by Fermi-LAT and MAGIC. We then use the measured SED and redshift information to characterize the radio to VHE broadband emission within a standard one-zone SSC scenario and investigate the origin of the detected variability. In this paper, we assume cosmological parameters $H_{0}=67 \mathrm{~km} \mathrm{~s}^{-1} \mathrm{Mpc}^{-1}$, $\Omega_{\mathrm{m}}=0.315, \Omega_{\Lambda}=0.685$ (Planck Collaboration XVI 2014).

\section{MAGIC observations and results}

\subsection{Observation and data analysis}

The MAGIC stereoscopic system consists of two IACTs with a mirror dish diameter of $17 \mathrm{~m}$ located at the Roque de los Muchachos, La Palma in Canary Island $\left(28.8^{\circ} \mathrm{N}, 17.8^{\circ} \mathrm{W}\right.$ at $2200 \mathrm{~m}$ a.s.1.). The MAGIC telescopes have been operating in stereoscopic mode since autumn 2009, which provided integral sensitivity of $0.76 \%$ of the Crab Nebula flux above $300 \mathrm{GeV}$ for $50 \mathrm{~h}$ observation time (Aleksić et al. 2012).

The object MAGIC J2001+439 was observed between 2009 November 7 and 26 for a total of 9.0 h. The MAGIC observations were also performed in a MWL campaign between 2010 July 6 and September 8 for a total of 14.4 h. The data were taken with zenith angles in the range $20 \mathrm{deg}-40 \mathrm{deg}$ in 2009 November and with zenith angles in the range $15 \mathrm{deg}-30 \mathrm{deg}$ during the campaign in 2010 July-September. The observations were carried out in wobble mode (Fomin et al. 1994), where the target source position has an offset of $0.4^{\circ}$ from the camera center. The direction of the wobble offset between two symmetric sky locations is alternated every $20 \mathrm{~min}$ to minimize systematic errors originating from possible exposure inhomogeneities.

The data were analyzed using the standard analysis chain (Aleksić et al. 2012) with the MAGIC Analysis and Reconstruction Software (MARS; Moralejo et al. 2009; Zanin et al. 2013). Camera images were cleaned using a sum imagecleaning method (Lombardi et al. 2011; Zanin 2011). This algorithm originated from the concept of the sum trigger (Rissi 2009; Haefner et al. 2011). In this procedure, the signals are clipped in amplitude and all possible combinations of 2, 3 and 4 neighboring pixels in the camera are summed up. If the sum of the charges is above a certain threshold within a short time interval, these pixels are considered to belong to the shower image. The clipping ensures that afterpulses or strong night sky background fluctuations do not dominate the summed pixels. Generally, the sum image-cleaning method recovers more pixels than the standard method. This is important for reconstructing shower images of low-energy gamma rays.

\subsection{Results}

Figure 1 shows the distribution of the squared angular distance $\left(\theta^{2}\right)$ between the reconstructed arrival directions of the events and the real source position in the camera.

We found an excess of events $N_{\text {ex }}=125.0 \pm 20.2$ in the energy range above $70 \mathrm{GeV}$ in the observation on 2010 July 16 in which the effective observation time was $1.36 \mathrm{~h}$ (see Fig. 1c). This gamma-ray excess yields a signal significance of $6.3 \sigma$ calculated using Eq. (17) of Li \& Ma (1983). When correcting for the seven observations (trials) performed in the MWL campaign, we find a post-trial signal significance of $6.0 \sigma$, hence implying the first detection of VHE gamma rays from 2 FGL J2001.1+4352. The time-averaged integral photon flux above $200 \mathrm{GeV}$ corresponds to $\sim 9 \%$ of the Crab Nebula flux. The detected position of the excess (RA(J2000): $\left.20.021 \pm 0.001 \mathrm{~h}, \operatorname{Dec}(\mathrm{J} 2000): 43.879 \pm 0.010^{\circ}\right)$ is consistent with the position of 2 FGL J2001. $1+4352^{3}$ within $0.02^{\circ}$. The distribution of the gamma-ray excess is consistent with a point-like source. The source was not detected during the rest of the observing campaign (see Fig. 1b). In the data between 2010 July and September (excluding 2010 July 16), the significance of the excess in $8.0 \mathrm{~h}$ of observations is $1.1 \sigma$ above the energy threshold of $70 \mathrm{GeV}$. Including the observations from 2010 July 16 , the significance (above $70 \mathrm{GeV}$ ) of the accumulated dataset is $4 \sigma$. The data collected in 2009 November led to $6.8 \mathrm{~h}$ of effective observation time, where we measure a gamma-ray excess above the energy threshold of $100 \mathrm{GeV}$ at a significance level of $1.8 \sigma$ (see Fig. 1a). The slightly higher energy threshold in the 2009 MAGIC observations with respect to that of the 2010

3 2FGL J2001.1+4352 is located at $\mathrm{RA}(\mathrm{J} 2000)=20.019 \mathrm{~h}$, $\operatorname{Dec}(\mathrm{J} 2000)=43.879^{\circ}$ in the 2 FGL catalog (Nolan et al. 2012). 


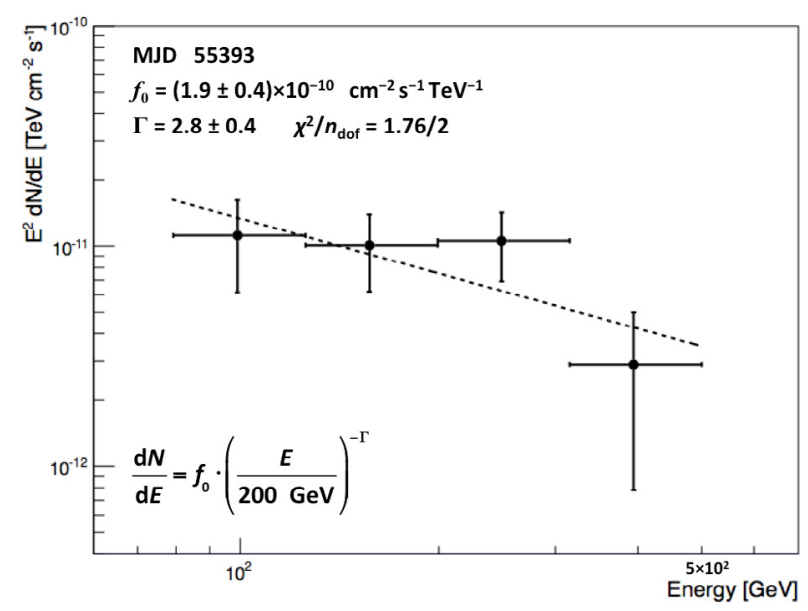

Fig. 2. VHE differential energy spectrum of MAGIC J2001+439 observed on 2010 July 16 with the MAGIC stereo system. The parameter values from the power-law fit are reported in the legend.

observations is due to the different zenith angle range for these two sets of observations.

The differential spectrum from the flare on 2010 July 16 can be described by a simple power law:

$\frac{\mathrm{d} N}{\mathrm{~d} E}=f_{0} \times\left(\frac{E}{200 \mathrm{GeV}}\right)^{-\Gamma}$

with flux normalization $f_{0}=(1.9 \pm 0.4) \times 10^{-10} \mathrm{~cm}^{-2} \mathrm{~s}^{-1} \mathrm{TeV}^{-1}$ and photon index $\Gamma=2.8 \pm 0.4$. The spectrum is fitted by a power-law function between 78 and $500 \mathrm{GeV}$. The systematic uncertainties in the spectral measurements with MAGIC stereo observations are $11 \%$ in the normalization factor (at $300 \mathrm{GeV}$ ) and $0.15-0.20$ in the photon index. The error on the flux does not include uncertainty on the energy scale. The energy scale of the MAGIC telescopes is determined with a precision of about $17 \%$ at low energies $(E<100 \mathrm{GeV})$ and $15 \%$ at medium energies $(E>300 \mathrm{GeV})$. Further details are reported in Aleksić et al. (2012). We corrected for our limited energy resolution and energy bias using the Tikhonov unfolding algorithm (Albert et al. 2007). The result is shown in Fig. 2.

\section{Multiband variability and correlations}

\subsection{Instrumentation and data analysis}

\subsubsection{Fermi-LAT}

The Fermi-LAT is a pair conversion telescope designed to cover the energy band from $20 \mathrm{MeV}$ to values greater than $300 \mathrm{GeV}$ (Atwood et al. 2009), which operates in survey mode. The data were analyzed with the Fermi Science Tools package version v9r27p1 available from the Fermi Science Support Center (FSSC) ${ }^{4}$. For this analysis, only events belonging to the Pass7 Source class and located in a circular region of interest of $10^{\circ}$ radius of $2 \mathrm{FGL} \mathrm{J2001.1+4352}$ were selected. Moreover, events with zenith angles greater than $100^{\circ}$ were removed to reduce the contamination from the Earth-limb gamma-rays, which are produced by cosmic rays interacting with the upper atmosphere, and time intervals during which the rocking angle of the spacecraft exceeded $52^{\circ}$ were excluded. The background model used to extract the gamma-ray signal includes a Galactic diffuse emission component and isotropic components

\footnotetext{
4 http://fermi.gsfc.nasa.gov/ssc
}

(including residual cosmic rays), which were modeled using the files gal_2yearp7v6_v0.fits and isotropic iso_p7v6source.txt that are publicly available ${ }^{5}$. The normalizations of the components comprising the total background model were allowed to vary freely during the spectral point fitting. The spectral fluxes were derived with the post-launch instruments response functions P7_V6_SOURCE and by applying an unbinned maximum likelihood technique (Mattox et al. 1996) to events in the energy range spanning $300 \mathrm{MeV}$ to $300 \mathrm{GeV}$. All the sources from the $2 \mathrm{FGL}$ catalog located within $10^{\circ}$ radius were included in the model of the region. The source position and initial spectrum parameters in the XML file were set to those of the 2FGL catalog. Flux upper limits at $95 \%$ confidence level were computed for those time intervals with a test statistic (TS; Mattox et al. 1996) value below four. The systematic uncertainty in the flux is dominated by the systematic uncertainty in the effective area, which is estimated as $10 \%$ at $100 \mathrm{MeV}$, is increased to $5 \%$ at $560 \mathrm{MeV}$, and is increased to $10 \%$ at $10 \mathrm{GeV}$ (Ackermann et al. 2012). The systematic uncertainties are smaller than the statistical uncertainties of the data points in the light curve and spectra.

\subsubsection{Swift}

Swift is equipped with three telescopes: the Burst Alert Telescope (BAT; Barthelmy et al. 2005), which covers the $15-150 \mathrm{keV}$ range, the X-ray telescope (XRT; Burrows et al. 2005), which covers the $0.3-10 \mathrm{keV}$ energy range, and the UltraViolet/Optical telescope (UVOT; Roming et al. 2005), which covering the $180-600 \mathrm{~nm}$ wavelength range with six bandpass filters.

The results reported here relate to measurements performed with XRT and UVOT. The BAT instrument is not sufficiently sensitive to detect this object: MAGIC J2001+439 is in neither the 70-month BAT catalog (Baumgartner et al. 2013) nor the BAT transient monitor paper (Krimm et al. 2013).

The Swift satellite observed the source 15 times in 2010. All XRT observations were carried out using the Photon Counting (PC) readout mode. The dataset was first processed with the XRTDAS software package (v.2.9.3) developed at the ASI Science Data Center (ASDC) and distributed by HEASARC within the HEASoft package (v. 6.15.1). Event files were calibrated and cleaned with standard filtering criteria with the xrtpipeline task using the calibration files available in the Swift CALDB version 20140120. The average spectra were extracted from the cleaned event files. Events for the spectral analysis were selected within a circle of 20 pixel $\left(\sim 46^{\prime \prime}\right)$ radius, which encloses about $90 \%$ of the point-spread function (PSF), centered on the source position. The background was extracted from a nearby circular region of 40 pixel radius. Two observations were excluded from the analysis due the very short exposure leading to insufficient number of counts. The ancillary response files (ARFs) were generated with the xrtmkarf task applying corrections for PSF losses and CCD defects using the cumulative exposure map. Before the spectral fitting, the $0.3-10 \mathrm{keV}$ source energy spectra were binned to ensure a minimum of 20 counts per bin. The results from the Swift-XRT observations during the MWL campaign in 2010 are summarized in Table A.1. The $\mathrm{X}$-ray count rates and hardness ratios for the intra-night light

\footnotetext{
5 http://fermi.gsfc.nasa.gov/ssc/data/access/lat/ BackgroundModels.html
} 


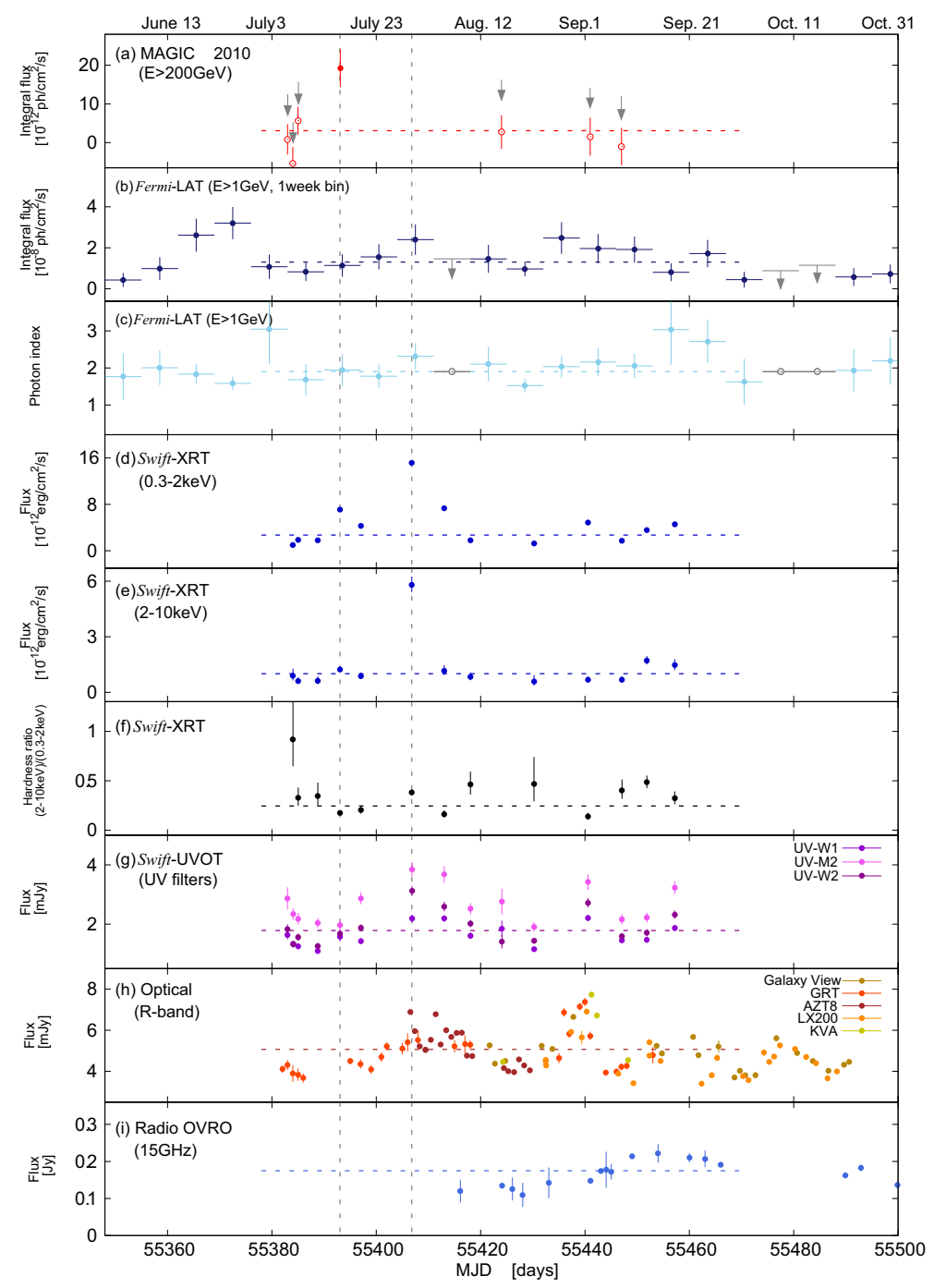

Fig. 3. Multiband light curves of MAGIC J2001+439 during the observing campaign in 2010. All light curves show fluxes from single night observations, except for Fermi-LAT. a) MAGIC light curve above $200 \mathrm{GeV}$. The red filled circle depicts the flux during the VHE flare on 2010 July 16 (the only significant detection), while the open circles correspond to flux points with excess significances between -1.3 and $1.6 \sigma$ (calculated according to Li \& Ma 1983, Eq. (17)). The gray arrows report the $95 \%$ confidence level upper limits, calculated using a photon index of 2.8. b) Fermi-LAT light curve above $1 \mathrm{GeV}$ with a weekly binning. The gray arrows report the flux upper limits at $95 \%$ confidence level, which were calculated (using the photon index of 1.9, reported for this source in the 2FGL catalog) for the time intervals with TS $<4$. c) Fermi-LAT photon index computed with a weekly binning. The gray open circles denote the assumed photon index for the calculation of the upper limits. d) Swift-XRT light curve in the energy range from 0.3 to $2 \mathrm{keV}$. e) Swift-XRT light curve in the energy range from 2 to $10 \mathrm{keV}$. f) Hardness ratio $(2-10 \mathrm{keV}) /(0.3-2 \mathrm{keV})$. g) SwiftUVOT light curves for the three UV filters. h) Optical $R$-band light curves from different telescopes (see legend). i) Radio light curve at $15 \mathrm{GHz}$ from the OVRO telescope. The horizontal dotted lines show the result of a fit with a constant function (the UV-W2 flux points were used for the Swift-UVOT light curve). The first and second gray vertical dashed lines denote July 16 and July 29 respectively. curve from July 16 (the day with the VHE flare) were extracted from the automatic Swift XRT analysis for Fermi-LAT sources ${ }^{6}$.

The Swift-UVOT observations on MG4 J200112+4352 were conducted with UV filters only (namely $W 1, M 2$, and $W 2$ ). We performed an aperture photometry analysis for all filters in all the observations using the standard UVOT software distributed within the HEAsoft 6.13 package and the calibration included in the version 20140120 of the Swift CALDB. Counts were first extracted from an aperture of $5^{\prime \prime}$ radius for all filters and converted to fluxes using the standard zero points (Poole et al. 2008). We excluded a nearby contaminating star from the aperture (USNO B1.0 1338-0359172, photographic magnitudes $\mathrm{B} 2=15.81, \mathrm{R} 2=14.98$; Monet et al. 2003), which is the only one within the extraction region in our UV images. We also tried the "curve of growth" method, which is included in the official software for apertures with FWHM radii for each filter. We obtained compatible results, except for larger errors using the second method. The fluxes were then de-reddened using $E(B-V)=0.498$ (Schlegel et al. 1998; Schlafly \& Finkbeiner $2011)$ and with $A_{\lambda} / E(B-V)$ ratios calculated for UVOT filters using the mean galactic interstellar extinction curve from (Fitzpatrick 1999). The results were also carefully checked for other possible contaminations. No intra-observation variability has been detected taking the nearby star into account. The results of Swift-UVOT are summarized in Table A.2. The results

\footnotetext{
${ }^{6}$ http://www. swift.psu.edu/monitoring
}

obtained for the individual observations show a peak in the source's broadband SED at the M2 frequency with the energy flux for the M2 filter being up to a factor of two larger than for the W1 filter. The energy flux for M2 is up to 50\% higher than that for W2. Comparable "narrow features" in the SED, which are not expected in regular synchrotron bumps from leptonic theoretical scenarios (see Figs. 13-15) are observed in the SwiftUVOT results for other sources (e.g. see Fig. 4 in Archambault et al. 2014), hence we conclude that it is an instrumental effect (not related to the object we are studying). This effect could be due to the source having a $B-V$ that is out of the validity range indicated by Poole et al. (2008) for their flux calibrations in the $U V$ bands. This instrumental effect, however, does not have any impact on the main results reported here.

\subsubsection{Optical band}

The optical $R$-band flux density was monitored during the campaign by several instruments. These optical aperture photometry observations were performed with the $35 \mathrm{~cm}$ optical telescope at the KVA observatory on La Palma (that operates in close collaboration with the MAGIC telescopes), the Goddard Robotic Telescope (GRT) at the Goddard Geophysical and Astronomical observatory, the Galaxy View observatory, a $70 \mathrm{~cm}$ AZT-8 telescope at the Crimean Astrophysical observatory, and a $40 \mathrm{~cm}$ LX-200 telescope in St. Petersburg observatory. All the observed 
$R$-band magnitudes were corrected with the Galactic extinction of $A_{\lambda}=1.219 \mathrm{mag}$ (Schlafly \& Finkbeiner 2011), but the optical data points shown in Fig. 3 are not host-galaxy corrected. The typical statistical error is $\sim 0.03 \mathrm{mag}$, which is comparable to the systematic error related to these measurements. For the typical fluxes measured during these observations (15.7 mag), the relative statistical error of the flux measurements is $\sim 3 \%$.

As reported in Sect. 3.3.2, the contribution from the host galaxy is small in comparison to the blazar emission. The overall host galaxy emission, when corrected for the Galactic extinction, would be $\sim 0.5 \mathrm{mJy}$. However, the contribution of the host galaxy to the measured blazar emission would depend on the details of the optical observations and data reduction. For the particular case of KVA (which used a fixed aperture radius of 4 arcsec for these observations), the host galaxy contribution to the measured blazar fluxes would be $\sim 0.3 \mathrm{mJy}$. Such estimates for the other telescopes were not performed, but one would expect similar values within $\sim 0.1 \mathrm{mJy}$. Therefore, the subtraction of the constant emission from the host galaxy would only shift the fluxes down by $\sim 6 \%$ with an additional difference among instruments at the level of $\sim 2 \%$. We considered these small offsets not essential for the results reported in this paper.

\subsubsection{Radio band}

Radio monitoring at $15 \mathrm{GHz}$ was performed with the $40 \mathrm{~m}$ telescope at the Owens Valley Radio Observatory (OVRO) through an ongoing blazar monitoring program ${ }^{7}$. Observations of MAGIC J2001+439 commenced on 2010 August 8 and were scheduled approximately twice per week. The observation and calibration procedures are described in detail in Richards et al. (2011). Flux densities were measured in a $3 \mathrm{GHz}$ wide band centered at $15.0 \mathrm{GHz}$, using off-axis dual beam optics with azimuth double switching to remove atmospheric and ground interference. The flux density scale is determined from regular observations of 3C 286 by assuming the value of $3.44 \mathrm{Jy}$ at $15.0 \mathrm{GHz}$ (Baars et al. 1977), which leads to about 5\% scale uncertainty and is not included in our error bars. Individual uncertainties are estimated from an error model that accounts for non-thermal random errors in addition to the measured scatter during the observation. The error model works well on average but occasionally produces excessively conservative uncertainties, particularly during poor weather. About $2 \%$ of the radio data was excluded due to bad weather.

\subsection{Results}

\subsubsection{Overall multiband light curves}

This MWL campaign was conducted over 2.5 months in 2010. The overall MWL light curves of MAGIC J2001+439 during the campaign are shown in Fig. 3. From all the MAGIC observations, only the one from 2010 July 16 yielded a significant detection. The other observations yielded excesses with a signal significance below $2 \sigma$. In the light curve, we show the photon fluxes and also the $95 \%$ confidence level flux upper limits calculated night by night during 2010 July to September. The upper limits were derived by assuming a power-law spectrum with a photon index of $\Gamma=2.8$, which is the one measured for the observation on 2010 July 16. We note that the computed upper limits depend on the assumed photon index. When using a photon index of $\Gamma=4.0$, the upper limits increase $\sim 5 \%$. The integral flux above $200 \mathrm{GeV}$ on 2010 July 16

\footnotetext{
7 http://www. astro.caltech.edu/ovroblazars
}

is $F_{200 \mathrm{GeV}}=(1.9 \pm 0.5) \times 10^{-11} \mathrm{ph} \mathrm{cm}^{-2} \mathrm{~s}^{-1}$, which corresponds to $\sim 9 \%$ the flux of the Crab Nebula. The integral flux upper limit during the non-detection period in 2009 November shows $F_{200 \mathrm{GeV}}<1.0 \times 10^{-11} \mathrm{ph} \mathrm{cm}^{-2} \mathrm{~s}^{-1}$ above $200 \mathrm{GeV}$. The FermiLAT light curve is plotted with a temporal bin width of one week in the energy range from 1 to $300 \mathrm{GeV}$. The one-week averaged Fermi-LAT photon index (for a power-law function above $1 \mathrm{GeV}$ ) was also computed (see third panel in Fig. 3), indicating the absence of spectral variability (on weekly timescales) during the three-month observing campaign.

The multiband light curves from Fig. 3 show that MAGIC $\mathbf{J} 2001+439$ is variable at all energy bands with the largest flux variations in the X-ray light curve. It is worth noting the $2-10 \mathrm{keV}$ X-ray flux during the VHE flare from July 16 is only twice as large as that measured during previous SwiftXRT observations in 2010 July, while the $2-10 \mathrm{keV}$ flux from July 29 (MJD 55 406) is five times higher than that of July 16. The $0.3-2 \mathrm{keV} \mathrm{X}$-ray light curve shows the same trend as the variation of the $2-10 \mathrm{keV} \mathrm{X}$-ray light curve, and the hardness ratio also changes in this campaign. Unfortunately, bad weather conditions precluded MAGIC observations on July 29. Moreover, the OVRO monitoring observations started soon after the announcement of the first VHE detection (Mariotti et al. 2010).

In Fig. 4, we report the long-term light curves at $\mathrm{GeV}$ gamma-rays, optical, and radio bands, as a result of a dedicated optical/radio follow-up during more than one year. The emission in these three energy bands shows a gradual decrease from 2010 through 2011. Further details on the long-term variability and correlations are discussed in Sects. 3.2.3 and 3.2.4.

\subsubsection{July 16 intranight light curves}

The intranight light curves of MAGIC J2001+439 on 2010 July 16 are shown in Fig. 5. The top panel shows the light curve of the MAGIC observations with an interval of $20 \mathrm{~min}$ in the energy range above $200 \mathrm{GeV}$. A constant fit to the data yielded a flux above $200 \mathrm{GeV}$ of $F_{200 \mathrm{GeV}}=(1.8 \pm 0.5) \times 10^{-11} \mathrm{ph} \mathrm{cm}^{-2} \mathrm{~s}^{-1}$ with a $\chi^{2} / n_{\text {d.o.f. }}=9.3 / 4$, which corresponds to a $\chi^{2}$ probability of $P_{\chi}=5.4 \%$. This indicates that the MAGIC light curve is consistent with a constant flux hypothesis within $95 \%$ confidence level. The Swift-XRT intranight light curve and the hardness ratio plots are shown in the same figure, respectively. The fit with a constant to the X-ray count rate gives a $\chi^{2}$ of 12.3 for $n_{\text {d.o.f. }}=11$ $\left(P_{\chi}=34 \%\right)$, and to the hardness ratio curve gives a $\chi^{2}$ of 2.9 for $n_{\text {d.o.f. }}=2\left(P_{\chi}=23 \%\right)$. We did not detect any statistically significant intra-night variability.

\subsubsection{Fractional variability}

To quantify the energy dependence of variability amplitudes, we computed the fractional variability amplitude of the light curves for each spectral band (Vaughan et al. 2003). The fractional variability amplitude $F_{\text {var }}$ is calculated as

$F_{\text {var }}=\sqrt{\frac{S^{2}-e^{2}}{F^{2}}}$

where $S^{2}$ is the total variance of the light curve, $e^{2}$ is the mean squared error, $F$ is the mean flux.

The uncertainty of $F_{\text {var }}$ is defined as

$\Delta F_{\mathrm{var}}=\sqrt{F_{\mathrm{var}}^{2}+\operatorname{err}\left(\sigma_{\mathrm{NXS}}^{2}\right)}-F_{\mathrm{var}}$,

as reported in Poutanen et al. (2008). 


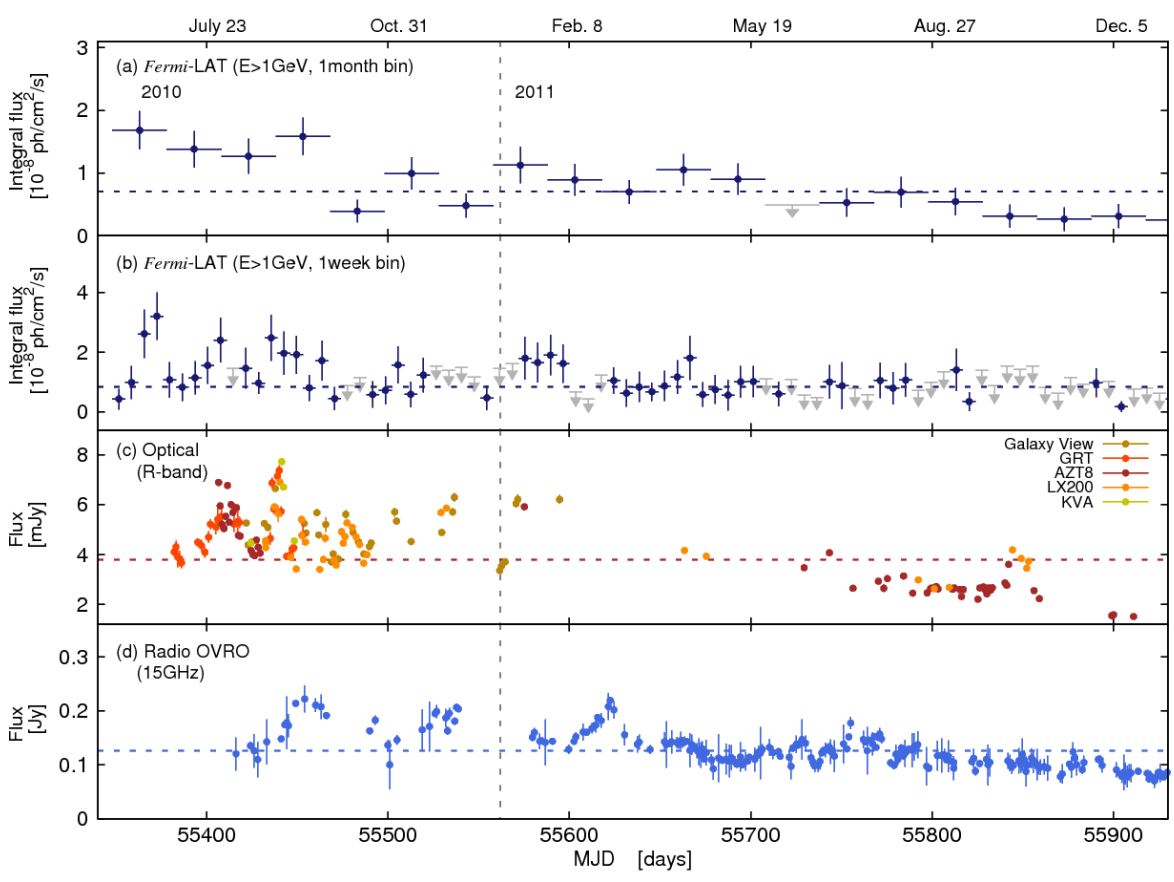

Fig. 4. Long-term light curves of MAGIC J2001+439 in 2010 and 2011. a) Fermi-LAT light curve above $1 \mathrm{GeV}$ with a monthly binning. b) Fermi-LAT light curve above $1 \mathrm{GeV}$ with a weekly binning. The gray arrows report the flux upper limits at $95 \%$ confidence level, which were calculated for the time intervals with TS $<4$. c) Optical $R$-band light curves, d) Radio light curve at $15 \mathrm{GHz}$. The horizontal dotted lines show the result of a fit with a constant function. See caption of Fig. 3 for further details.
The $\operatorname{err}\left(\sigma_{\mathrm{NXS}}^{2}\right)$, which is the error in the normalised excess (NXS) variance, is given by Eq. (11) of Vaughan et al. (2003)

$$
\operatorname{err}\left(\sigma_{\mathrm{NXS}}^{2}\right)=\sqrt{\left(\sqrt{\frac{2}{N}} \frac{e^{2}}{F^{2}}\right)^{2}+\left(\sqrt{\frac{e^{2}}{N}} \frac{2 F_{\mathrm{var}}}{F}\right)^{2}} .
$$

This methodology to quantify the variability has some caveats. The fractional variability $F_{\text {var }}$ is determined for the temporal bin and the source sampling from the light curves used, and the source variability might actually depend on that. In other words, a densely sampled light curve with very small temporal bins might allow us to see flux variations that are hidden otherwise, and hence we might obtain a larger $F_{\text {var }}$. In the set of light curves shown in Figs. 3 and 4, one can see that some energy bands are better sampled than others. In particular, the information from the gamma-ray band is limited with only seven observations performed with MAGIC and fluxes on weekly/monthly time intervals (instead of daily time intervals) obtained with Fermi-LAT. Another caveat is that the fractional variability given by Eq. (2) expects data points with similar error bars (within one dataset). A few data points with substantially (by factors of a few) larger error bars would have a larger impact in $e^{2}$ than in $S^{2}$, hence biasing $F_{\text {var }}$ towards lower values. In our multi-instrument dataset, the band that is most affected by this effect is the $15 \mathrm{GHz}$ radio light curve provided by OVRO. Despite the above-mentioned caveats, the $F_{\text {var }}$ from Vaughan et al. (2003) is a useful methodology to quantify in a simple way the variability in the different energy bands sampled during this observing MWL campaign.

Figure 6 shows the fractional variability obtained with the data reported in the light curves from Figs. 3 and 4 . The obtained $F_{\text {var }}$ values for all energy bands are listed in Table 1 . During the MWL campaign in summer 2010, we measured significant $F_{\text {var }}$ values for the optical, UV, X-ray, and VHE gamma-ray bands with the variability being greatest at X-ray and VHE. The $F_{\text {var }}$ at VHE is dominated by the large flux increase during the flaring activity on July 16 , although this $F_{\text {var }}$ value needs to be taken with caveats due to the small number of observations in comparison with those performed at other energy bands and the lack of
Table 1. Fractional variability amplitudes for different energy bands and two time periods.

\begin{tabular}{lc}
\hline \hline Energy bands & $F_{\mathrm{var}} \pm \Delta F_{\mathrm{var}}$ \\
\hline (campaign in 2010) & $1.92 \pm 0.50$ \\
MAGIC $(E>200 \mathrm{GeV})$ & $0.14 \pm 0.17$ \\
Fermi-LAT one-week bins $(E>1 \mathrm{GeV})$ & $1.03 \pm 0.05$ \\
Swift-XRT $(2-10 \mathrm{keV})$ & $0.90 \pm 0.01$ \\
Swift-XRT $(0.3-2 \mathrm{keV})$ & $0.28 \pm 0.02$ \\
Swift-UVOT (W2) & $0.22 \pm 0.02$ \\
Swift-UVOT (M2) & $0.22 \pm 0.02$ \\
Swift-UVOT (W1) & $0.19 \pm 0.01$ \\
Optical $(R$-band) & $0.16 \pm 0.06$ \\
Radio $(15 \mathrm{GHz})$ & \\
\hline (long-term in 2010-2011) & $0.48 \pm 0.07$ \\
Fermi-LAT one-month bins $(E>1 \mathrm{GeV})$ & $0.26 \pm 0.10$ \\
Fermi-LAT one-week bins $(E>1 \mathrm{GeV})$ & $0.30 \pm 0.01$ \\
Optical $(R$-band) & $0.21 \pm 0.01$ \\
Radio $(15 \mathrm{GHz})$ & \\
\hline
\end{tabular}

VHE observations during the strong X-ray flare on July 29. As for the long-term behavior, we measure significant $F_{\text {var }}$ values in the three bands sampled, namely radio, optical and HE gammarays. The fractional variability obtained for these three bands is similar.

\subsubsection{Multiband correlations}

We quantified the correlation among the MWL light curves shown in Figs. 3 and 4 by applying the Discrete Correlation Function (DCF) technique from Edelson \& Krolik (1988) to investigate the correlation between different energy bands for different time lags. For each of these pairs, we can compute the unbinned discrete correlation functions (UDCF),

$U D C F_{i j}=\frac{\left(F_{a_{i}}-F_{a}\right)\left(F_{b_{j}}-F_{b}\right)}{\sqrt{\left(S_{a}^{2}-e_{a}^{2}\right)\left(S_{b}^{2}-e_{b}^{2}\right)}}$,

where $F_{a_{i}}$ and $F_{b_{j}}$ are the data pair in the bin associated with the pairwise time lag $\Delta t_{i j}=t_{j}-t_{i}, F_{a}$ and $F_{b}$ are the mean flux 


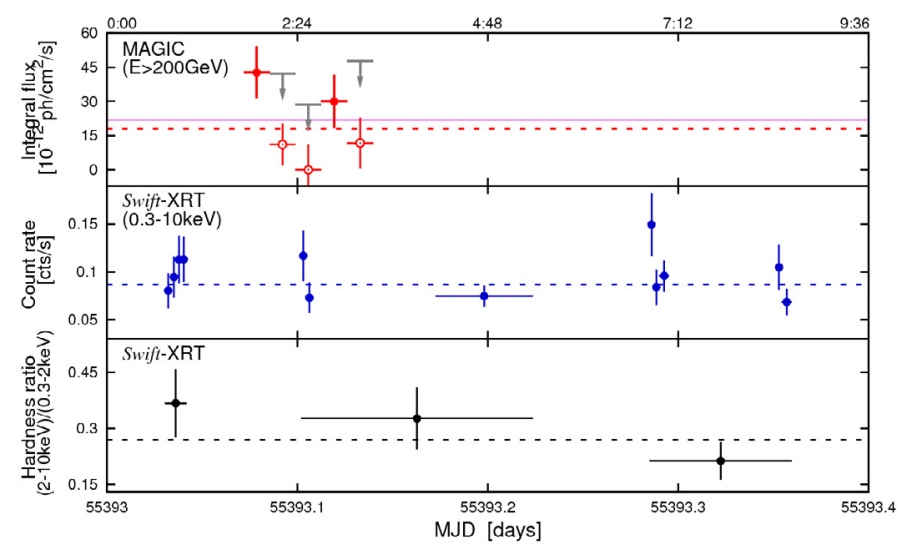

Fig. 5. Intra-night multiband light curves of MAGIC J2001+439 observations on 2010 July 16. Top panel: VHE gamma-ray flux above $200 \mathrm{GeV}$, as measured by MAGIC. The open circles depict the fluxes with excess significances between 0 and $1.2 \sigma$ (calculated according to Li \& Ma 1983, Eq. (17)). The gray arrows report the $95 \%$ confidence level upper limits. The magenta solid line depicts $10 \%$ of the Crab Nebula flux, while the red dashed line reports the result of a fit with a constant function $\left(\chi^{2} / n_{\text {d.o.f. }}=9.3 / 4\right)$. Middle panel: X-ray count rate in the energy range $0.3-10 \mathrm{keV}$, as measured by Swift XRT. The blue dashed line depicts the result of a fit with a constant function $\left(\chi^{2} / n_{\text {d.o.f. }}=\right.$ 12.3/11). Bottom panel: hardness ratio $(2-10 \mathrm{keV}) /(0.3-2 \mathrm{keV})$. The black dashed line depicts the result of a fit with a constant function $\left(\chi^{2} / n_{\text {d.o.f. }}=2.9 / 2\right)$. The X-ray count rate and hardness ratios were extracted from the automatic Swift XRT analysis for LAT sources ${ }^{6}$.

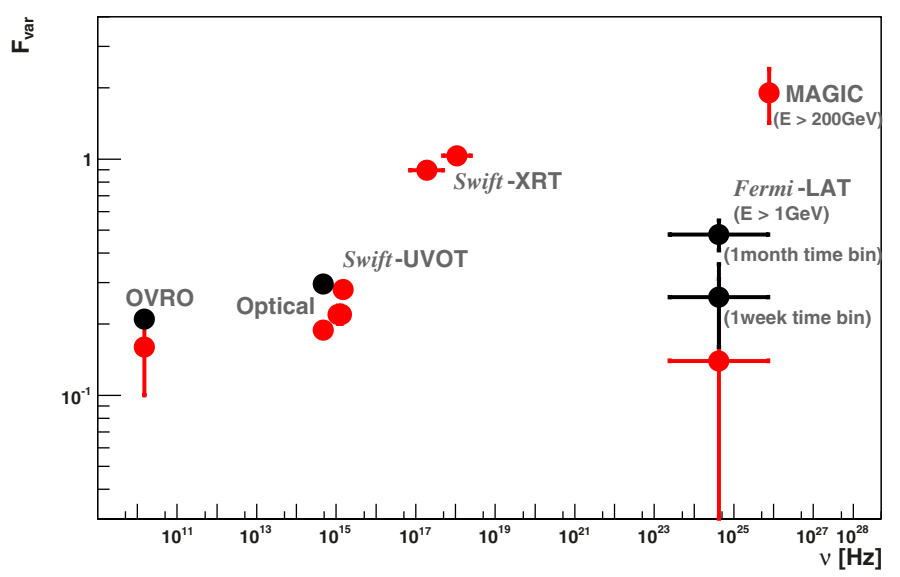

Fig. 6. Fractional variability vs. energy. The red data points refer to the MWL campaign light curves in 2010 July to September reported in Fig. 3, while the black data points refer to the long-term light curves in 2010 to 2011 reported in Fig. 4.

values, $S_{a}$ and $S_{b}$ are the standard deviations, and $e_{a}^{2}$ and $e_{b}^{2}$ are the mean measurement errors squared, respectively.

The DCF for a given time lag of $\tau$ is then constructed as

$D C F(\tau)=\frac{1}{M} \sum_{i j} U D C F_{i j}\left(\Delta t_{i j}\right)$,

where the sum runs over the $M$ pairs of observations separated by $\tau-\Delta \tau / 2 \leq \Delta t_{i j} \leq \tau+\Delta \tau / 2$, where $\Delta \tau$ is the chosen bin width.

The uncertainty on the value of the DCF in a given bin is calculated as the RMS variance of all the contributing $U D C F_{i j}$ about the value $D C F(\tau)$,

$\sigma_{\mathrm{DCF}}(\tau)=\frac{1}{M-1}\left(\sum\left[U D C F_{i j}-D C F(\tau)\right]^{2}\right)^{\frac{1}{2}}$.
We investigated the correlation among the different bands shown in Fig. 3, obtaining a significant correlation only for the UV vs. optical band. Figure 7 shows that the DCF for time lags of \pm 30 days for the UV (W2) and optical $(R)$ band. We used 5 -day bins for the time lags, which minimizes the impact of the 2-3 day time gaps in the UV data. The plots show clearly that the significant correlation occurs only for a time lag zero. The Pearson's correlation coefficient ${ }^{8}$ for these two datasets (depicted in the right panel in Fig. 7) is $r=0.90$ with an accidental probability $P$ of no-correlation ${ }^{8}$ of $0.04 \%$ (with $n_{\text {d.o.f. }}=8$ ). The significant correlation is not surprising, given the proximity of these two energy bands.

The DCF plots for long-term light curves from Fig. 4 are reported in Fig. 8. The three panels show an overall positive correlation for all time lags, which is produced by the clear long-term decrease in the radio/optical/gamma-ray fluxes over many month timescales. The DCF for Fermi vs. radio and Fermi vs. optical cover \pm 0.5 years with 30 -day time lag bins. The large error bars in the DCF values, which are caused by the relatively large error bars in the gamma-ray fluxes, preclude the investigation of any temporal structure in the correlation.

The DCF for the optical vs. radio flux cover time lags from -50 days to +120 days with a temporal bin of 5 days. The asymmetry is driven by the result that the radio observations started about one month after the optical observations, and they extend further in time than the optical observations. This means that there is a lower tolerance to apply negative time shifts (i.e. the optical light curve is shifted to earlier times) and a higher tolerance to apply positive time shifts (i.e. the optical light curve is shifted to later times). Given the large number of data points and the relatively small single-night measurement errors, the errors in the DCF values are small, which indicates a temporal structure on the top of the overall positive correlation. The DCF is highest in the time lag range from 0 to +50 days with a maximum value at about +40 days. To investigate this DCF peak at about 40 days, we computed the normalized optical and radio long-term light curves, where the data flux values of each measurement are divided by the mean flux of the entire light curve. There are some structures in the optical and radio light curves that are similar in amplitude and are better aligned when shifting the optical light curve by +40 days; yet, there are also several other structures, which occur in one band and not in the other. Moreover, we note that +40 days is the minimum time lag needed to get the optical light curve starting at the same day as the radio light curve. Given the different length and density of observations for these two bands, we cannot make definite conclusions about the temporal structure observed in the DCF for these two bands, apart from the positive correlation produced by the long-term decrease in the light curves. Further studies would require more homogeneous and better sampled light curves.

Figure 9 shows flux-flux linear correlation plots of time lag zero for long-term light curves. The derived Pearson's correlation coefficients are $r=0.59$ for the $\mathrm{GeV}$ gamma-ray vs. optical $R$-band, $r=0.43$ for the $\mathrm{GeV}$ gamma-ray vs. radio, and $r=0.48$ for the radio vs. the optical $R$-band. The accidental probabilities are $P=2.0 \%$ (with number of degrees of freedom $n_{\text {d.o.f. }}=13$ ), $P=10.0 \%$ (with $n_{\text {d.o.f. }}=14$ ), and $P=0.3 \%$ (with $n_{\text {d.o.f. }}=35$ ) for $\mathrm{GeV} /$ optical, $\mathrm{GeV} /$ radio and radio/optical, respectively. These values indicate that there is a marginally significant positive linear correlation among the GeV/optical bands, and a much more significant positive correlation for the

\footnotetext{
8 This is a standard formulation that one can find in Sect. 14.5 of Press et al. (1992).
} 

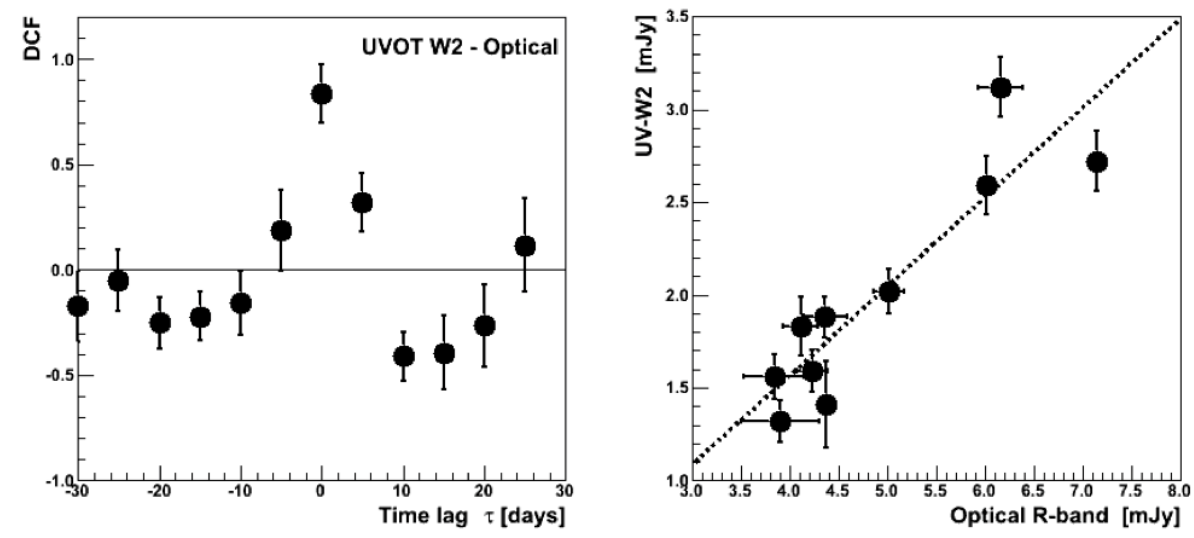

Fig. 7. Discrete correlation function for different time lags computed for the UV (W2) and optical $(R)$ data obtained during the MWL campaign in 2010 and the flux linear correlation plot for a time lag of zero. The dotted line shows the best fit with a linear function.
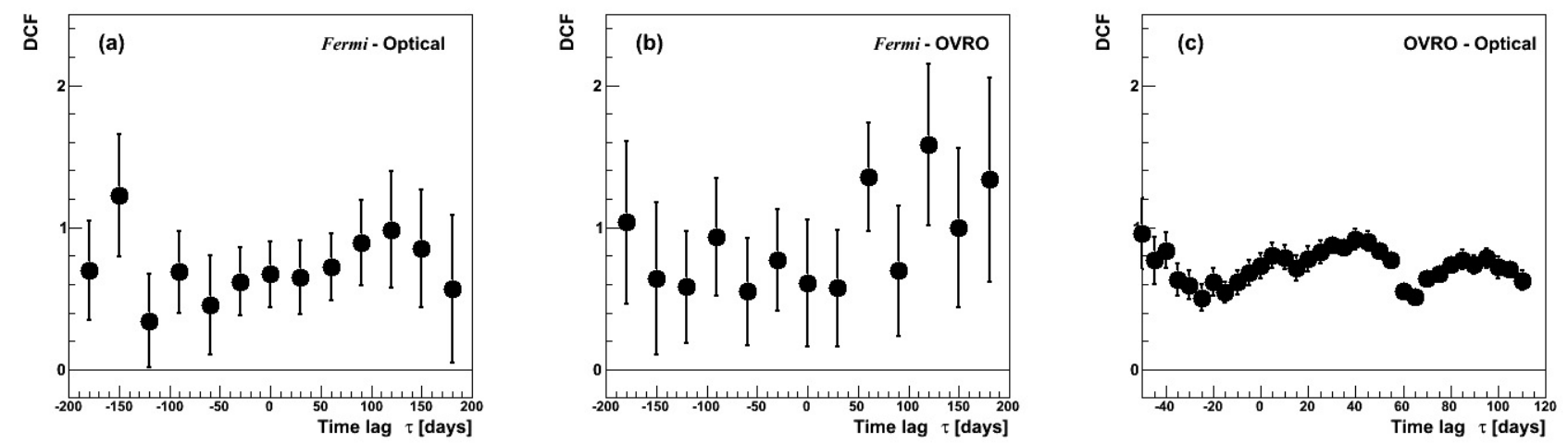

Fig. 8. Discrete correlation function for different time lags for the long-term light curves from Fig. 4. a) One-month-averaged Fermi-LAT fluxes $(E>1 \mathrm{GeV})$ vs. one-month-averaged optical $R$-band fluxes. b) One-month-averaged Fermi-LAT fluxes $(E>1 \mathrm{GeV})$ vs. one-month-averaged radio $15 \mathrm{GHz}$ fluxes. c) Radio $15 \mathrm{GHz}$ fluxes vs. optical $R$-band fluxes (only single observations occurring during the same day were used).
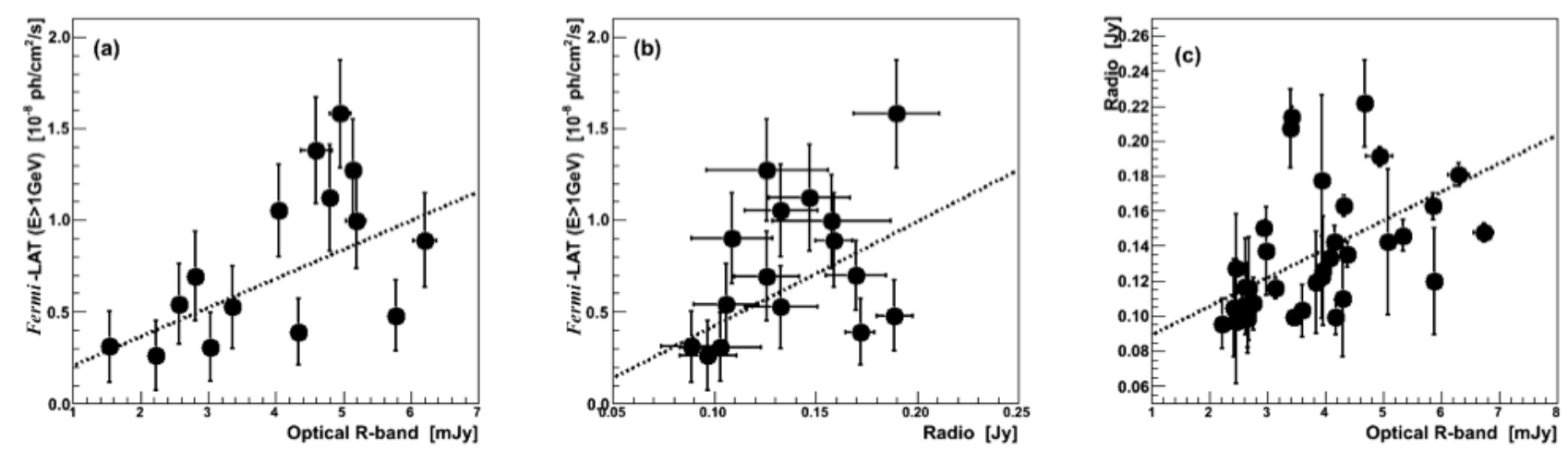

Fig. 9. Flux-flux linear correlation plots for a time lag of zero for the long-term light curves reported in Fig. 4. a) One-month-averaged Fermi-LAT fluxes $(E>1 \mathrm{GeV})$ vs. one-month-averaged optical $R$-band fluxes. b) One-month-averaged Fermi-LAT fluxes $(E>1 \mathrm{GeV})$ vs. one-monthaveraged radio $15 \mathrm{GHz}$ fluxes. c) Radio $15 \mathrm{GHz}$ fluxes vs. optical $R$-band fluxes. (Only single observations occurring during the same day were used.) The dotted line shows the best fit with a linear function.

radio/optical bands. The higher significance for the correlation between the radio/optical bands is due to the larger number of measurements and smaller uncertainties in the measured fluxes (as implied by formulae (5)-(7)).

\subsection{Redshift measurement of the blazar MAGIC J2001+439}

Since the redshift of MAGIC J2001+439 was still uncertain, we used two independent methods to determine it.

\subsubsection{Redshift estimation using the gamma-ray spectrum}

The redshift of a gamma-ray source can be estimated (or at least constrained) using the measured gamma-ray spectra, once a particular EBL model is assumed. In this work, we adopted the EBL model from Franceschini et al. (2008). If we assume that the intrinsic source gamma-ray spectrum can be expressed by a simple power-law function $\mathrm{d} N / \mathrm{d} E \propto E^{-\Gamma_{\text {int }}}$, where the fitted intrinsic photon index is $\Gamma_{\text {int }}$, one can set upper limits on the source redshift under the assumption that the intrinsic source gammaray spectrum cannot be harder than 1.5 . This limit is physically motivated from shock acceleration arguments, as discussed in Aharonian et al. (2006). As shown in Fig. 10, when taking the uncertainty in the measured VHE spectrum with MAGIC into account, we find that this assumption yields an upper limit on the redshift of $z<0.6$ with a $95 \%$ confidence level.

Another estimate on the redshift can be obtained using the measured gamma-ray spectra with Fermi and MAGIC, as 


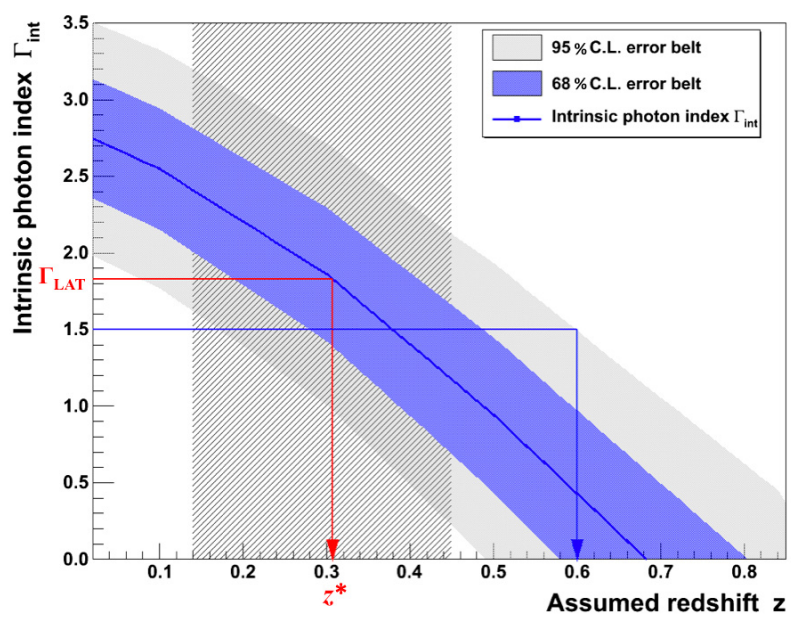

Fig. 10. Intrinsic photon index $\Gamma_{\text {int }}$ as a function of the redshift assuming the EBL model from Franceschini et al. (2008). The blue and gray filled areas correspond to the $1 \sigma(68 \%)$ and $2 \sigma(95 \%)$ confidence error belts, respectively. The red arrow indicates the estimated redshift $z^{*}$, which is determined by the redshift value at which $\Gamma_{\text {int }}=\Gamma_{\mathrm{LAT}}=1.83 \pm 0.18$. The diagonal shaded area shows the error range of $z^{*}$, where the error takes both the errors on the MAGIC VHE photon index and the FermiLAT HE photon index into account. See text for calculation of the reconstructed redshift $z_{\text {rec }}$ using the value $z^{*}$. The blue arrow indicates the $95 \%$ confidence level upper limit on the redshift, which is obtained when $\Gamma_{\text {int }}=1.5$, within the $2 \sigma(95 \%)$ confidence error belt.

reported in Prandini et al. (2011). We analyzed Fermi-LAT data from MAGIC J2001+439 between 2010 July 1 and August 1 and obtained a one-month-averaged Fermi-LAT spectrum. This spectrum has a spectral index of $\Gamma_{\mathrm{LAT}}=1.83 \pm 0.18$, when being characterized by a power-law function in the energy range between $300 \mathrm{MeV}$ and $30 \mathrm{GeV}$. The integral flux is $F_{300 \mathrm{MeV}}=(3.9 \pm 0.8) \times 10^{-8} \mathrm{ph} \mathrm{cm}^{-2} \mathrm{~s}^{-1}$ in the energy range above $300 \mathrm{MeV}$. We define the redshift of $z^{*}$ by requiring that the power-law index of the observed VHE gamma-ray spectrum, when corrected for the EBL absorption, is equal to the powerlaw index of $\Gamma=1.83$ observed by Fermi-LAT at energies which are not affected by the EBL absorption. As shown in Fig. 10, this procedure leads to $z^{*}=0.31 \pm 0.16$. In this calculation, we did not consider instrumental systematic errors in the determination of the spectral indices from MAGIC and Fermi-LAT. We followed the prescription given in Prandini et al. (2011) with the difference that, we also considered the uncertainty in the power-law index from the HE spectrum measured with Fermi, in addition to the uncertainty in the power-law index from the VHE spectrum when computing the uncertainty in the parameter $z^{*}$. Therefore, our uncertainty in the parameter $z^{*}$ is larger, but more reliable than the one that would have been derived following Prandini et al. (2011). To determine the reconstructed redshift $z_{\text {rec }}$, we used the empirical relation reported in Prandini et al. (2011) that relates the true redshifts of known distance sources $z_{\text {true }}$ with their $z^{*}$ values, $z^{*}=A+B z_{\text {true }}$, where $A=0.036 \pm 0.014$ and $B=1.60 \pm 0.14$. Using this prescription, we obtain a reconstructed redshift $z_{\mathrm{rec}}=0.17 \pm 0.10$. The systematic uncertainty related to this method is estimated to be 0.05 in the redshift value.

\subsubsection{Redshift determination using the measured flux of the host galaxy}

The MAGIC J2001+439 (MG4 J200112+4352) was observed by the NOT telescope on 2013 June 13 to study the host galaxy.
To increase the detection probability, the observations were timed to coincide with an optical low state $(R \sim 16.8$, corresponding to $F \sim 1.8 \mathrm{mJy}$ ) of the target. We obtained nine images, each with 900 s exposure time, through the I-band filter using the ALFOSC instrument equipped with a $2048 \times$ 2048 E2V chip with a gain factor of $0.327 e^{-} /$Analog to Digital Units (ADU), and readout noise of $4.2 e^{-}$. The total field of view of ALFOSC is $6.5 \times 6.5$, and the pixel scale is $0 .^{\prime} 19 /$ pixel. The transparency of the atmosphere remained constant for all the observations, which allowed for photometric measurements. The images were bias-subtracted and flat-fielded with twilight flats, after which the fringe pattern was removed using an archival fringe map. Individual images were then registered using 13 stars over the field of view and co-added. The resulting image has a total exposure time of $2 \mathrm{~h} 15 \mathrm{~min}$ and FWHM of 0.72 .

The calibration of the field was obtained from $I$-band observations of MAGIC J2001+439 and BL Lac in photometric conditions with the 72 " Perkins Telescope at Lowell Observatory on 2013 November 2 and 3. We first used the BL Lac comparison star sequence in Fiorucci \& Tosti (1996) to calibrate five stars in the field of MAGIC J2001+439, and the same stars were then used to calibrate the NOT image. The uncertainty of this calibration is $0.05 \mathrm{mag}$. As a crosscheck, we performed a second calibration using the $N$-magnitudes from 25 nearby stars from GSC2.3 and found a difference of $17 \pm 9 \%$ between these two calibrations.

To study the host galaxy, we fitted two-dimensional surface brightness models to the observed light distribution of MAGIC $\mathrm{J} 2001+439$. Details of this process can be found in Nilsson et al. (1999, 2003). In short, we first determined the background level around MAGIC J2001+439 and a nearby star S1 (Fig. 11) by removing the background tilt and then measuring empty sky regions around the targets. Next, we determined the PSF from two field stars located 55 arcsec and 92 arcsec away from MAGIC $\mathrm{J} 2001+439$. These fields were carefully selected not to be contaminated by foreground/background stars and roughly equal to MAGIC J2001+439 in peak intensity. The two field stars were close enough to have the same PSF. We then fitted the PSF to MAGIC J2001+439 and S1 and subtracted the resulting model, which accurately removed the star S1 (showing a good fit with the PSF model) but revealed a clear excess around MAGIC J2001+439. The latter was then fitted with a model consisting of an unresolved nucleus and a host galaxy, represented by the Sérsic (1968) profile with Sérsic index $n=4$ (de Vaucouleurs profile). The number of free parameters in this fit was nine: position and magnitude of the nucleus $\left(x_{n}, y_{n}, m_{n}\right)$, host galaxy position $\left(x_{\mathrm{g}}, y_{\mathrm{g}}\right)$, magnitude $\left(m_{\mathrm{g}}\right)$, effective radius $\left(r_{\mathrm{e}}\right)$, ellipticity $(\epsilon)$, and position angle $(\mathrm{PA})$, which is defined counter-clockwise from the North. The fit was performed using pixels within 5.7 arcsec from the center of MAGIC J2001+439, excluding any pixels affected by overlapping targets and subtracting S1 prior to the fit.

The results of these fits are summarized in Figs. 11 and 12 and Table 2 . In addition to the best-fit values, we give the errors of the fitted parameters $\left(\sigma_{\text {fit }}\right)$ and the calibration error $\left(\sigma_{\text {cal }}\right)$ in Table 2. The former were estimated with $\sim 100$ Monte Carlo simulations of the fit, which included the effects of photon noise, readout noise background uncertainty, and PSF variability (see Nilsson et al. 2003, for details). We were not able to obtain a perfect fit (reduced $\chi^{2}=3.03$ ) mainly due to the PSF mismatch in the core of MAGIC J2001+439 and noise in the PSF wings, both of which were not included in the noise model. However, our simulations include these effects and show that the results are not biased in any way due to not achieving a reduced $\chi^{2}=1.0$. 


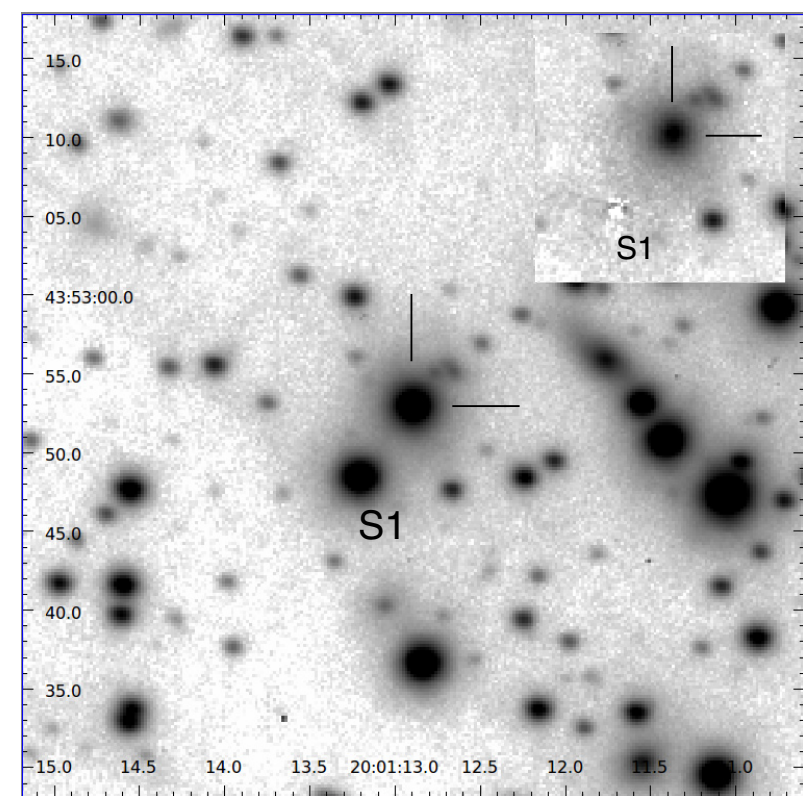

Fig. 11. Portion of the NOT $I$-band image. Field size is $50^{\prime \prime} \times 50^{\prime \prime}$. North is up, and east is to the left. MAGIC J2001+439 and a nearby star (S1) are indicated. The inset shows MAGIC J2001+439 and S1 after subtracting a properly scaled PSF, showing the host galaxy more clearly.

The redshift of MAGIC J2001+439 was estimated using the observed host galaxy magnitude $I=17.15 \pm 0.06$ and the result by Sbarufatti et al. (2005) that the luminosities of BL Lac host galaxies are confined to a relatively narrow range of $M_{R}=$ $-22.8 \pm 0.5$. We used $R-I=0.7$, leading to $M_{I}=-23.5$, the K-correction from Fukugita et al. (1995), and the evolution correction $E(z)=0.84 * z$ to iteratively determine the redshift consistent with $I=17.15$ and $M_{I}=-23.5$. For the galactic extinction, we used the value in NED, $A_{I}=0.846$, which is based on the dust reddening study by Schlafly \& Finkbeiner (2011). The redshift was estimated 1000 times with each time drawing $I$, $M_{I}$, and $A_{I}$ from a Gaussian distribution with standard deviations of $0.06,0.5$, and 0.14 , respectively. The resulting $z$ distribution is roughly Gaussian with average $z=0.18$ and standard deviation $\sigma_{z}=0.04$. It should be noted that almost all uncertainty in this $z$ estimate arises from the relatively broad distribution of $M_{I}$.

A potential bias (systematic error) in this estimation could come from the assumption of the true BL Lac host galaxy luminosity distribution. In this respect, Shaw et al. (2013) studied the host galaxies of 475 Fermi BL Lacs and obtained an average host galaxy luminosity of $M_{R}=-22.5$, which is $0.3 \mathrm{mag}$ fainter than the flux $M_{R}=-22.8 \pm 0.5$ used here (retrieved from Sbarufatti et al. 2005). If we used $M_{R}=-22.5$ in our calculation, we would obtain $z=0.16 \pm 0.04$, which is well within the statistical uncertainties of our measurement. Moreover, it should be stressed that Shaw et al. (2013) reported that their result may be biased because they studied targets, which had no host detections at the time of their study, which means that they were probably selecting targets with fainter hosts (the brighter hosts were already detected by earlier authors).

The two redshift measurements reported in Sects. 3.3.1 and 3.3.2 $(z=0.17 \pm 0.10$ and $z=0.18 \pm 0.04)$ are compatible and consistent with the rough estimate reported by Bassani et al. (2009), and the lower limit reported by Shaw et al. (2013). The second method (using the optical measurement of the host galaxy) is more reliable because it uses less assumptions and
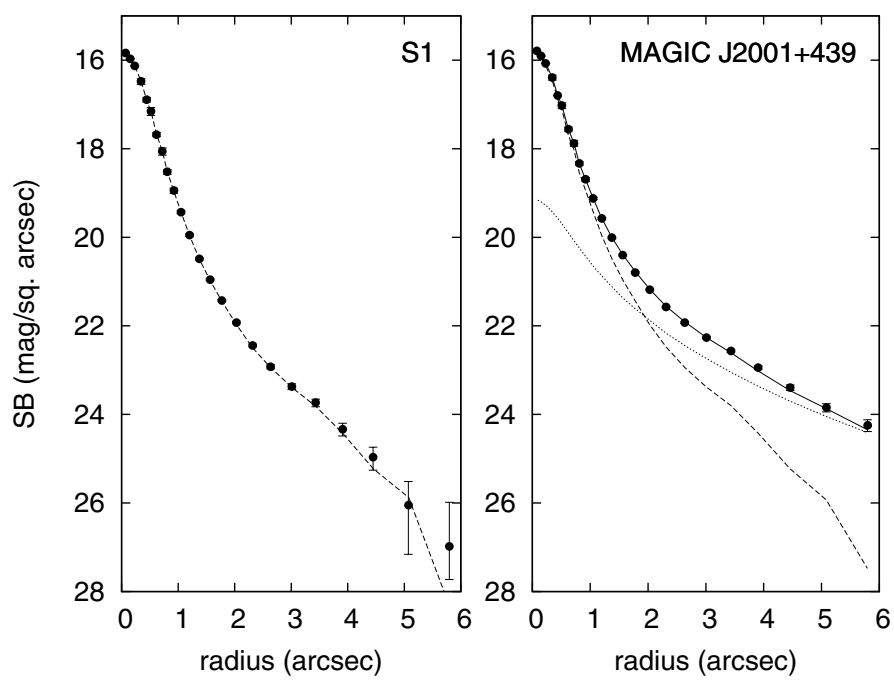

Fig. 12. Surface brightness profiles of star S1 and MAGIC J2001+439 with model profiles: nucleus + host galaxy (solid line), nucleus (dashed), and host galaxy (dotted).

Table 2. Host galaxy fit results.

\begin{tabular}{llll}
\hline \hline Parameter & Value & $\sigma_{\text {fit }}$ & $\sigma_{\text {cal }}$ \\
\hline Nucleus magnitude $m_{n}$ & 16.08 & 0.02 & 0.05 \\
Host magnitude $m_{\mathrm{g}}$ & 17.15 & 0.04 & 0.05 \\
Host effective radius $r_{\mathrm{e}}$ & $2^{\prime \prime} 4$ & $0^{\prime} .4$ & \\
Host ellipticity $\epsilon$ & 0.15 & 0.03 & \\
Host PA & $178^{\circ}$ & $3^{\circ}$ & \\
\hline
\end{tabular}

Notes. $\sigma_{\text {fit }}$ gives the statistical error of each parameter as determined by error simulations, and $\sigma_{\text {cal }}$ is the calibration error (see the text for further details).

yields a smaller uncertainty in the redshift value. We use $z=$ 0.18 throughout the rest of this paper.

\section{Discussion}

In Fig. 13, we show the simultaneous MWL SED of MAGIC J2001+439 on 2010 July 16. The MAGIC data points show the deabsorbed spectrum with a redshift of $z=0.18$ using the EBL model of Franceschini et al. (2008). The deabsorbed spectrum is compatible with a simple power law with photon index $\Gamma=2.3 \pm 0.4$. A one-zone SSC model, as described in the appendix of Takami (2011) was used to interpret the MWL SED. In this model, the emission region is assumed to be spherical with radius $R$ and to be filled by a tangled magnetic field of intensity $B$ in a comoving frame. The emission region is in motion with a Lorentz factor of $\Gamma$ and a viewing angle of $\theta$ in the observer frame. The injected energy distribution of the relativistic emitting electrons is described by an unsmoothed broken powerlaw function ${ }^{9}$,

$N(\gamma)= \begin{cases}K \gamma^{-n_{1}} & \left(\gamma_{\min }<\gamma<\gamma_{\mathrm{bk}}\right) \\ K \gamma_{\mathrm{bk}}^{n_{2}-n_{1}} \gamma^{-n_{2}} & \left(\gamma_{\mathrm{bk}}<\gamma<\gamma_{\max }\right),\end{cases}$

where $K$ is the normalization factor of the electron density, extending from $\gamma_{\min }$ to $\gamma_{\max }$ with indices $n_{1}$ and $n_{2}$ below and above

9 The code described in Takami (2011) can parameterize the electron energy distribution with both a smoothed and unsmoothed broken power law. 

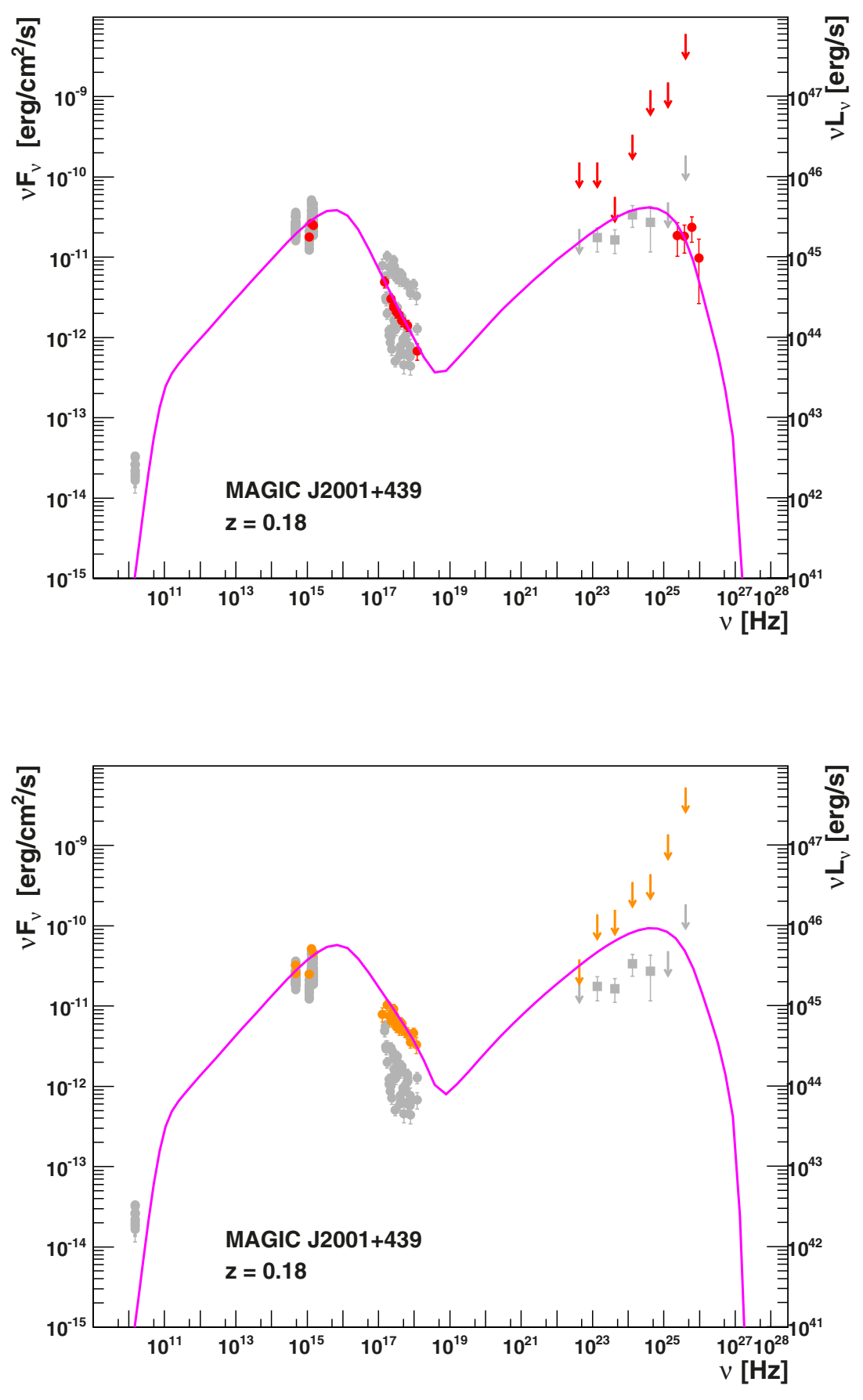

Fig. 13. Broadband SED for 2010 July 16. The simultaneous data are depicted with red filled circles (which involve observations from MAGIC, FermiLAT, Swift-XRT, and Swift-UVOT). The gammaray data points were corrected for attenuation on the EBL using a redshift $z=0.18$ and the model from Franceschini et al. (2008). The red arrows show the 95\% upper limits for a one-day-averaged Fermi-LAT spectrum (MJD: 55 392.5-55 393.5), which is coincident with the MAGIC VHE observation from July 16 . The gray filled squares represent the one-month-averaged Fermi-LAT spectrum in 2010 July (MJD: 55 378-55 409), and the gray filled circles show all the radio/optical/UV/X-ray data taken during the MWL campaign in 2010, excluding July 16 . The magenta solid curve represents the resulting intrinsic spectrum parameterized with a one-zone SSC model. See text for further details.

Fig. 14. Broadband SED for 2010 July 29. The simultaneous data are depicted with orange filled circles (which involve observations from Fermi-LAT, Swift-XRT, Swift-UVOT and optical $R$-band). The gamma-ray data points were corrected for attenuation on the EBL using a redshift $z=0.18$ and the model from Franceschini et al. (2008). The orange arrows show the $95 \%$ upper limits for a oneday-averaged Fermi-LAT spectrum (MJD: 55 40655407 ) coincident with the Swift observations from July 29. The gray filled squares represent the onemonth-averaged Fermi-LAT spectrum in 2010 July (MJD: 55 378-55 409), and the gray filled circles show all the radio/optical/UV/X-ray data taken during the MWL campaign in 2010, excluding July 29. The magenta solid curve represents the resulting intrinsic spectrum parameterized with a one-zone SSC model. See text for further details.

the break Lorentz factor $\gamma_{\mathrm{bk}}$, respectively. Relativistic effects are taken into account by the Doppler factor $\delta=[\Gamma(1-\beta \cos \theta)]^{-1}$. We obtained the following one-zone SSC scenario parameters: $\gamma_{\min }=1.0, \gamma_{\mathrm{bk}}=3.9 \times 10^{4}, \gamma_{\max }=6.0 \times 10^{5}, n_{1}=2.0, n_{2}=4.8$, $K=5.2 \times 10^{3} \mathrm{~cm}^{-3}, B=55 \mathrm{mG}, R=20.4 \times 10^{15} \mathrm{~cm}$ and $\delta=27$, where we used the redshift $z=0.18$, which is the value derived from the dedicated measurement reported in Sect. 3.3.2. The parameters $\gamma_{\min }$ and $n_{1}$ had been initially set to 1 and 2.0 , respectively. The estimated synchrotron emission peak of MAGIC $\mathrm{J} 2001+439$ is located at a high frequency $\sim 10^{16} \mathrm{~Hz}$, which indicates that this object is a typical HBL. The simultaneous MWL SED of MAGIC J2001+439 on 2010 July 16 can be described well by a one-zone SSC scenario.
Figure 14 shows the SED of the X-ray flare on 2010 July 29 with the simultaneous data from Fermi-LAT, Swift-XRT/UVOT, and optical $R$-band. There are no VHE gamma-ray observations due to bad weather at the MAGIC site. The X-ray spectrum from July 29 with a photon index of $2.5 \pm 0.1$ seems to be harder than that from July 16 , for which we obtained a photon index of $2.9 \pm 0.2$. The synchrotron component in the energy band between radio and X-rays shows significant variability (see Fig. 3). We also find a simultaneous increase of the UV energy flux during the X-ray flare. Therefore, we tried to parameterize the SED from July 29 by changing few parameters (with respect to the model used for July 16) by describing the electron spectrum, while keeping the environmental parameters constant of 
Table 3. One-zone SSC model parameters for the three different SEDs reported in Figs. 13-15.

\begin{tabular}{lcccccccccc}
\hline \hline State & $\gamma_{\min }$ & $\begin{array}{c}\gamma_{\mathrm{bk}} \\
{\left[10^{4}\right]}\end{array}$ & $\begin{array}{c}\gamma_{\max } \\
{\left[10^{5}\right]}\end{array}$ & $n_{1}$ & $n_{2}$ & $\begin{array}{c}K \\
{\left[10^{3} \mathrm{~cm}^{-3}\right]}\end{array}$ & $\begin{array}{c}B \\
{[\mathrm{mG}]}\end{array}$ & $\begin{array}{c}R \\
{\left[10^{15} \mathrm{~cm}\right]}\end{array}$ & $\delta$ & $z$ \\
\hline July 16 (VHE flare detection) & 1.0 & 3.9 & 6.0 & 2.0 & 4.8 & 5.2 & 55 & 20.4 & 27 & 0.18 \\
July 29 (X-ray flare) & 1.0 & 3.9 & 6.0 & 2.0 & 4.3 & 7.3 & 55 & 20.4 & 27 & 0.18 \\
Typical emission during the campaign & 1.0 & 2.8 & 6.0 & 2.0 & 4.8 & 5.2 & 55 & 20.4 & 27 & 0.18 \\
\hline
\end{tabular}

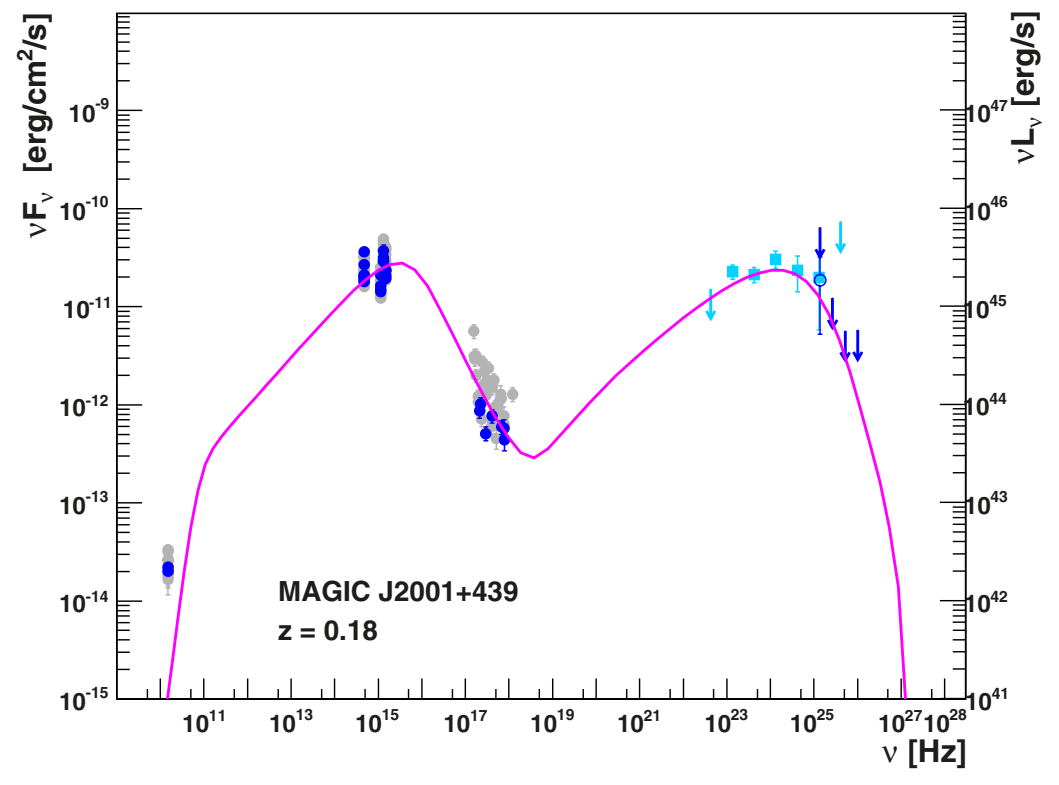

Fig. 15. Contemporaneous broadband SED for the low state during the MWL campaign in 2010. The blue open circle depicts the energy flux computed using all the MAGIC observations during the MWL campaign in 2010, after excluding the data during the VHE flare from 2010 July 16. This MAGIC data point contains a gamma-ray excess with a signal significance (calculated using Eq. (17) of Li \& Ma 1983) of 1.4 $\sigma$. The blue arrows report the $95 \%$ confidence level energy flux upper limits derived from the MAGIC observations. The cyan filled squares represent the averaged Fermi-LAT spectrum from the MWL campaign in 2010 (MJD: 55382 55458). The cyan arrows report the Fermi-LAT 95\% confidence level energy flux upper limits for the energy bins with TS $<4$. The gamma-ray data points and upper limits were corrected for attenuation on the EBL using a redshift $z=0.18$ and the model from Franceschini et al. (2008). The blue filled circles show the Swift-XRT, Swift-UVOT, optical, and radio data obtained during the days when MAGIC observed the source (with the exception of July 16). The gray filled circles show all the radio/optical/UV/X-ray data taken during the MWL campaign in 2010, excluding July 16 and July 29 . The magenta solid curve represents the resulting intrinsic spectrum parameterized with a one-zone SSC model. See text for further details. the model. The obtained one-zone SSC model parameters that reproduce the observed SED data are summarized in Table 3. The increase and hardening of the X-ray spectrum is parameterized by an increase in the electron number density ( $K$ increased from $5.2 \times 10^{3} \mathrm{~cm}^{-3}$ to $7.3 \times 10^{3} \mathrm{~cm}^{-3}$ ) and a hardening in the slope of the electron spectrum above the break energy $\left(n_{2}\right.$ changed from 4.8 to 4.3 ). This could be interpreted as an injection of fresh relativistic electrons into the emission region, which should also cause a higher flux in the gamma-ray bands. According to this theoretical scenario, both the synchrotron and the SSC bumps went up substantially during this X-ray flare. The observational data can only confirm the large increase in the synchrotron bump. However, we also note that such a change in the SED could have been produced by alternative scenarios, such as a change in the magnetic field strength. More higher quality gamma-ray data would be required to discriminate between different scenarios.

Figure 15 shows the contemporaneous broadband SED for the 2.5 month-long MWL campaign. The figure shows both the MAGIC spectrum energy flux point with a significance (per data point) below $2 \sigma$ and $95 \%$ confidence level upper limits, which were calculated assuming a power-law spectrum with a photon index of $\Gamma=2.8$. We analyzed the 2.5 months-averaged Fermi-LAT spectrum during the MWL campaign in 2010 (which corresponds to the periods of the Swift observations). The Fermi-LAT spectrum between $300 \mathrm{MeV}$ and $100 \mathrm{GeV}$ can be characterized by a power-law function with a spectral index of $\Gamma_{\text {LAT }}=2.02 \pm 0.08$. The integral flux above $300 \mathrm{MeV}$ is $F_{300 \mathrm{MeV}}=(5.0 \pm 0.6) \times 10^{-8} \mathrm{ph} \mathrm{cm}^{-2} \mathrm{~s}^{-1}$. To describe the measured SED with the one-zone SSC model, we had to lower the value of $\gamma_{\mathrm{bk}}$ with respect to the one used to describe the SED from July 16. Within this theoretical scenario, this indicates that, the energy distribution of relativistic electrons extended to higher energies in July 16, when the VHE gamma-ray flare was detected with MAGIC.

\section{Conclusions}

We performed a detailed study of the broadband emission of 2FGL J2001.1+4352 (previously named 0FGL J2001.0+4352), which had been associated to the bright radio source MG4 J200112+4352 in Bassani et al. (2009) and identified as a promising VHE emitter by the Fermi-LAT collaboration in 2009 October. We characterized the radio to VHE SED and quantified the multiband variability and correlations over short (few days) and long (many months) timescales.

The planned MAGIC observations led to the first VHE detection of this object, which we named MAGIC J2001+439. The multi-instrument observations showed variability in all the energy bands with the highest amplitude of variability in the X-ray and VHE bands. This source was significantly detected at VHE only during a $1.3 \mathrm{~h}$ long MAGIC observation on 2010 July 16. The time-averaged VHE spectrum during this night can be described by a power-law function from $78 \mathrm{GeV}$ to $500 \mathrm{GeV}$ with a differential photon index $\Gamma=2.8 \pm 0.4$ and a flux normalization at $200 \mathrm{GeV} f_{0}=(1.9 \pm 0.4) \times 10^{-10} \mathrm{~cm}^{-2} \mathrm{~s}^{-1} \mathrm{TeV}^{-1}$, which gives about $9 \%$ the flux of the Crab Nebula above $200 \mathrm{GeV}$. During the other nights, the VHE flux was lower than $5 \%$ the 
Crab Nebula flux. Besides the variability on few-day timescales, the long-term monitoring of MAGIC J2001+439 showed that the gamma-ray, optical, and radio emission gradually decreased on few-month timescales from 2010 through 2011, indicating that the overall radio, optical, and gamma-ray emission is produced (at least a fraction of it) in a single region by the same population of particles. A similar positively correlated trend in the $\mathrm{GeV} /$ optical and the radio/optical bands has been observed on other blazars monitored over many years (e.g. Aleksić et al. 2014a,b).

For the first time, we also determined, the redshift of this BL Lac object through the measurement of its host galaxy during low blazar activity. Because the luminosities of BL Lac host galaxies are confined to a relatively narrow range (Sbarufatti et al. 2005), we obtained $z=0.18 \pm 0.04$. Moreover, we used the Fermi-LAT and MAGIC gamma-ray spectra to provide an independent redshift estimation (Prandini et al. 2011) by obtaining $z=0.17 \pm 0.10$. The redshift values computed with these two independent methods are compatible within the quoted errors. The first method is more reliable because it uses fewer assumptions and yields a smaller uncertainty in the redshift value.

We studied the radio-to-VHE SEDs for three periods: 2010 July 16 when the source was significantly detected at VHE with MAGIC; 2010 July 29 when a large X-ray flux was measured (with no simultaneous VHE observations), and the entire dataset from the MWL campaign in summer 2010 with the exclusion of the observations from July 16 and July 29. Using our redshift measurement of $z=0.18$, we described the three broadband SEDs with a one-zone SSC model. The model parameters that we used are at the boundary of the SSC parameter distribution derived for a TeV blazar sample by Tavecchio et al. (2010). Within this theoretical scenario, we explain the changes in the broadband SEDs observed during the flaring activity in July 16 as produced by an extension of the electron energy distribution towards higher energies (increase in the parameter $\gamma_{\mathrm{bk}}$ ) and in the SED observed during the large X-ray flare on July 29 as produced by an increase in the number of electrons (increase in the parameter $K$ ) and a hardening of the high-energy tail of the electron energy distribution (hardening of the parameter $n_{2}$ ).

This new VHE detection adds one more BL Lac object, MAGIC J2001+439, to the short list of extragalactic VHE sources ${ }^{1}$. Moreover, the redshift measurements we performed determined that this is a relatively distant VHE BL Lac object. The characterization of the broadband SED with simultaneous observations during various activity levels is relevant in understanding the physical properties of the various blazar types and in finally moving towards AGN unification schemes.

Acknowledgements. We would like to thank the anonymous referee for providing detailed and constructive remarks that helped us to improve the manuscript. We would like to thank the Instituto de Astrofísica de Canarias for the excellent working conditions at the Observatorio del Roque de los Muchachos in La Palma. The support of the German BMBF and MPG, the Italian INFN, the Swiss National Fund SNF, and the Spanish MICINN is gratefully acknowledged. This work was also supported by the CPAN CSD2007-00042 and MultiDark CSD2009-00064 projects of the Spanish Consolider-Ingenio 2010 program, by grant 127740 of the Academy of Finland, by the DFG Cluster of Excellence "Origin and Structure of the Universe", by the DFG Collaborative Research Centers SFB823/C4 and SFB876/C3, and by the Polish MNiSzW grant 745/NHESS-MAGIC/2010/0. The Fermi-LAT Collaboration acknowledges support from a number of agencies and institutes for both development and the operation of the LAT as well as scientific data analysis. These include NASA and DOE in the United States, CEA/Irfu and IN2P3/CNRS in France, ASI and INFN in Italy, MEXT, KEK, and JAXA in Japan, and the K. A. Wallenberg Foundation, the Swedish Research Council and the National Space Board in Sweden. Additional support from INAF in Italy and CNES in France for science analysis during the operations phase is also gratefully acknowledged. We gratefully acknowledge the entire Swift team, the duty scientists and science planners for the ded- icated support, making these observations possible. The data on this paper are based partly on observations made with the Nordic Optical Telescope, operated by the Nordic Optical Telescope Scientific Association at the Observatorio del Roque de los Muchachos, La Palma, Spain, of the Instituto de Astrofisica de Canarias. We would like to thank T. Pursimo for performing the NOT observations used for the redshift determination. Part of the data were obtained with ALFOSC, which is provided by the Instituto de Astrofisica de Andalucia (IAA) under a joint agreement with the University of Copenhagen and NOTSA. The St.Petersburg University team acknowledges support from the Russian RFBR foundation, grant 12-02-00452. The authors thank Svetlana Jorstad for kindly providing the optical calibration images. The OVRO $40 \mathrm{~m}$ monitoring program is supported in part by NSF grants AST-0808050 and AST-1109911 and NASA grants NNX08AW31G and NNX11AO43G. T.H. was supported by the Jenny and Antti Wihuri foundation. The National Radio Astronomy Observatory is a facility of the National Science Foundation operated under cooperative agreement by Associated Universities, Inc. Special thanks to Richard Schwartz, who performed observations with the Galaxy View observatory and diligently reduced the data while fighting against a pancreatic cancer during the last months of his life.

\section{References}

Abdo, A. A., Ackermann, M., Ajello, M., et al. 2009, ApJS, 183, 46 Ackermann, M., Ajello, M., Albert, A., et al. 2012, ApJS, 203,4

Ackermann, M., Ajello, M., Allafort, A., et al. 2013, ApJS, 209, 34

Aharonian, F., Akhperjanian, A. G., Bazer-Bachi, A. R., et al. 2006, Nature, 440 1018

Albert, J., Aliu, E., Anderhub, H., et al. 2007, Nucl. Instr. Meth. A, 583, 494

Aleksić, J., Alvarez, E. A., Antonelli, L. A., et al. 2012, Astropart. Phys., 35, 435

Aleksić, J., Ansoldi, S., Antonelli, L. A., et al. 2014a, A\&A, 564, A5

Aleksić, J., Ansoldi, S., Antonelli, L. A., et al. 2014b, A\&A, 567, A135

Archambault, S., Aune, T., Behera, B., et al. 2014, ApJ, 785, L16

Atwood, W. B., Abdo, A. A., Ackermann, M., et al. 2009, ApJ, 697, 1071

Baars, J. W. M., Genzel, R., Pauliny-Toth, I. I. K., \& Witzel, A. 1977, A\&A, 61, 99

Band, D. L., \& Grindlay, J. E. 1985, ApJ, 298, 128

Barthelmy, S. D., Barbier, L. M., Cummings, J. R., et al. 2005, Space Sci. Rev., 120,143

Bassani, L., Landi, R., Masetti, N., et al. 2009, MNRAS, 397, 55

Baumgartner, W. H., Tueller, J., Markwardt, C. B., et al. 2013, ApJS, 207, 19

Berger, K., Paneque, D., \& Giavitto, G. 2010, in Proc. 5th Texas Symp. on Relativistic Astrophysics, TEXAS2010 (SLAC-PUB-15014)

Berger, K., Giavitto, G., Lindfors, E., et al. 2011, in Proc. Fermi Symp., C110509 [arXiv: $1110.6368 \mathrm{v} 1]$

Bloom, S. D., \& Marscher, A. P. 1996, ApJ, 461, 657

Burrows, D. N., Hill, J. E., Nousek, J. A., et al. 2005, Space Sci. Rev., 120 , 165

Condon, J. J., Cotton, W. D., Greisen, E. W., et al. 1998, AJ, 115, 1693

Dermer, C. D., \& Schlickeiser, R. 1993, ApJ, 416, 458

Domínguez, A., Primack, J. R., Rosario, D. J., et al. 2011, MNRAS, 410, 2556

Edelson, R. A., \& Krolik, J. H. 1988, ApJ, 333, 646

Fazio, G. G., \& Stecker, F. W. 1970, Nature, 226, 135

Finke, J. D., Razzaque S., \& Dermer, C. D. 2010, ApJ, 712, 238

Fiorucci, M., \& Tosti, G. 1996, A\&AS, 116, 403

Fitzpatrick, E. L. 1999, PASP, 111, 63

Fomin, V. P., Stepanian, A. A., Lamb, R. C., et al. 1994, Astropart. Phys., 2, 137

Franceschini, A., Rodighiero, G., \& Vaccari, M. 2008, A\&A, 487, 837

Fukugita, M., Shimasaku, K., \& Ichikawa, T. 1995, PASP, 107, 945

Gilmore, R. C., Madau, P., Primack, J. R., et al. 2009, MNRAS, 399, 1694

Gould, R. J., \& Schreder, G. 1966, Phys. Rev. Lett., 16, 252

Haefner, D., Schweizer, T., Dazzi, F., \& Corti, D., 2011, in Proc. 32nd ICRC, Beijing, 9, 246

Hauser, M. G., \& Dwek, E. 2001, ARA\&A, 39, 249

Kneiske, T. M., \& Dole, H. 2010, A\&A, 515, A19

Krimm, H. A., Holland, S. T., Corbet, R. H. D., et al. 2013, ApJS, 209, 14

Li, T., \& Ma, Y. 1983, ApJ, 272, 317

Lombardi, S., Berger, K., Colin, P., Ortega, A. D., \& Klepser, S. 2011, in Proc. 32nd ICRC, Beijing, 8, 183

Mannheim, K., \& Biermann, P. L. 1992, A\&A, 253, 21

Maraschi, L., Ghisellini, G., \& Celotti, A. 1992, ApJ, 397, 5

Mariotti, M. (the MAGIC Collaboration) 2010, ATel, 2753

Mattox, J. R., Bertsch, D. L., Chiang, J., et al. 1996, ApJ, 461, 396

Monet, D. G., Levine, S. E., Canzias, B., Ables, H. D., et al. 2003, AJ, 125, 984

Moralejo, A., Gaug, M., Carmona, E., et al. 2009, in Proc. 31st ICRC, Lodz [arXiv:0907.0943]

Mücke, A., Protheroe, R. J., Engel, R., et al. 2003, Astropart. Phys., 18, 593 
Nilsson, K., Pursimo, T., Takalo, L. O., et al. 1999, PASP, 111, 1223

Nilsson, K., Pursimo, T., Heidt, J., et al. 2003, A\&A, 400, 95

Nolan, P. L., Abdo, A. A., Ackermann, M., et al. 2012, ApJS, 199, 31

Planck Collaboration XVI, 2014, A\&A, 571, A16

Poole, T. S., Breeveld, A. A., Page, M. J., et al. 2008, MNRAS, 383, 627

Poutanen, J., Zdziarski, A. A., \& Ibragimov, A. 2008, MNRAS, 1427, 389

Prandini, E., Bonnoli, G., Maraschi, L., Mariotti, M., \& Tavecchio, F. 2010, MNRAS, 405, 76

Prandini, E., Bonnoli, G., Maraschi, L., Mariotti, M., \& Tavecchio, F. 2011, in Proc. Cosmic Radiation Fields: sources in the early Universe, CRF2010 [arXiv: 1101.5005]

Press, W. H., Teukolsky, S. A., Vetterling, W. T., \& Flannery, B. P. 1992, Numerical Recipes in C, The Art of Scientific Computing, 2nd edn.

Richards, J., Max-Moerbeck, W.; Pavlidou, V., et al. 2011, ApJS, 194, 29

Rissi, M. 2009, Ph.D. Thesis, ETH Zurich, Diss. ETH No. 18322

Roming, P. W. A., Kennedy, T. E., Mason, K. O., et al. 2005, Space Sci. Rev., 120,95

Sbarufatti, B., Treves, A., \& Falomo, R. 2005, ApJ, 635, 173

Schlafly, E. F., \& Finkbeiner, D. P. 2011, ApJ, 737, 103

Schlegel, D. J., Finkbeiner, D. P., \& Davis, M. 1998, ApJ, 500, 525

Sérsic, J. L. 1968, Atlas de galaxias australes (Cordoba: Observatorio Astronomico)

Shaw, M. S., Romani, R. W., Cotter, G., et al. 2013, ApJ, 764, 135

Sikora, M., Begelman, M. C., \& Rees, M. J. 1994, ApJ, 421, 153

Stecker, F. W. 1969, AJ, 157, 507

Stecker, F. W., Malkan, M. A., \& Scully, S. T. 2006, ApJ, 648, 774

Takami, H. 2011, MNRAS, 413, 1845

Tavecchio, F., Ghisellini, G., Ghirlanda, G., Foschini, L., \& Maraschi, L. 2010, MNRAS, 401, 1570

Urry, C. M., \& Padovani, P. 1995, PASP, 107, 803

Vaughan, S., Edelson, R., Warwick, R. S., \& Uttley, P., 2003, MNRAS, 345, 1271

Zanin, R. 2011, Ph.D. Thesis, Universitat Autonoma de Barcelona

Zanin, R., Carmona, E., Sitarek, J., Colin, P., et al. 2013, in Proc. 33rd ICRC, Rio de Janeiro, 773

IFAE, Campus UAB, 08193 Bellaterra, Spain

2 Università di Udine, and INFN Trieste, 33100 Udine, Italy

3 INAF National Institute for Astrophysics, 00136 Rome, Italy

4 Università di Siena, and INFN Pisa, 53100 Siena, Italy

5 Croatian MAGIC Consortium, Rudjer Boskovic Institute, University of Rijeka and University of Split, 10000 Zagreb, Croatia

6 Max-Planck-Institut für Physik, 80805 München, Germany

7 Universidad Complutense, 28040 Madrid, Spain

8 Inst. de Astrofísica de Canarias, 38200 La Laguna, Tenerife, Spain

9 University of Łódź, 90236 Lodz, Poland

10 Deutsches Elektronen-Synchrotron (DESY), 15738 Zeuthen, Germany

11 ETH Zurich, 8093 Zurich, Switzerland

12 Universität Würzburg, 97074 Würzburg, Germany

13 Centro de Investigaciones Energéticas, Medioambientales y Tecnológicas, 28040 Madrid, Spain

14 Technische Universität Dortmund, 44221 Dortmund, Germany

15 Inst. de Astrofísica de Andalucía (CSIC), 18080 Granada, Spain
16 Università di Padova and INFN, 35131 Padova, Italy

17 Università dell'Insubria, Como, 22100 Como, Italy

18 Unitat de Física de les Radiacions, Departament de Física, and CERES-IEEC, Universitat Autònoma de Barcelona, 08193 Bellaterra, Spain

19 Institut de Ciències de 1'Espai (IEEC-CSIC), 08193 Bellaterra, Spain

20 Japanese MAGIC Consortium, Division of Physics and Astronomy, Kyoto University, Japan

21 Finnish MAGIC Consortium, Tuorla Observatory, University of Turku and Department of Physics, University of Oulu, Finland

22 Inst. for Nucl. Research and Nucl. Energy, 1784 Sofia, Bulgaria

23 Universitat de Barcelona, ICC, IEEC-UB, 08028 Barcelona, Spain

24 Università di Pisa, and INFN Pisa, 56126 Pisa, Italy

25 Now at NASA Goddard Space Flight Center, Greenbelt, MD 20771, USA and Department of Physics and Department of Astronomy, University of Maryland, College Park, MD 20742, USA

26 Now at École polytechnique fédérale de Lausanne (EPFL), Lausanne, Switzerland

27 Now at Department of Physics \& Astronomy, UC Riverside, CA 92521, USA

28 Now at Finnish Centre for Astronomy with ESO (FINCA), Turku, Finland

29 Also at INAF-Trieste, Italy

30 Also at Instituto de Fisica Teorica, UAM/CSIC, 28049 Madrid, Spain

31 Now at Stockholm University, Oskar Klein Centre for Cosmoparticle Physics, 10691 Stockholm, Sweden

32 Now at GRAPPA Institute, University of Amsterdam, $1098 \mathrm{XH}$ Amsterdam, The Netherlands

33 INAF Istituto di Radioastronomia, 40129 Bologna, Italy

34 Dipartimento di Fisica e Astronomia, via Ranzani 1, 40127 Bologna, Italy

35 Cahill Center for Astronomy and Astrophysics, California Institute of Technology, 1200 E California Blvd, Pasadena, CA 91125, USA

36 Isaac Newton Institute of Chile, St. Petersburg Branch, St. Petersburg, Russia

37 Pulkovo Observatory, 196140 St. Petersburg, Russia

38 Astronomical Institute, St. Petersburg State University, St. Petersburg, Russia

39 National Radio Astronomy Observatory, PO Box 0, Socorro, NM 87801, USA

40 Agenzia Spaziale Italiana (ASI) Science Data Center, 00133 Roma, Italy

41 Department of Physics, Purdue University, 525 Northwestern Ave, West Lafayette, IN 47907, USA

42 Department of Physics and Mathematics, College of Science and Engineering, Aoyama Gakuin University, 5-10-1 Fuchinobe, Chuoku, Sagamihara-shi, 252-5258 Kanagawa, Japan

43 University of Missouri-St. Louis, St. Louis, Missouri, USA

44 Physics Department, California Polytechnic State University, San Luis Obispo, CA 94307, USA 


\section{Appendix A: Swift-XRT and Swift-UVOT results}

Table A.1. Results of Swift-XRT observation during MWL campaign 2010.

\begin{tabular}{lcccccc}
\hline \hline Observation date & $\begin{array}{c}\text { MJD } \\
\text { [days] }\end{array}$ & $\begin{array}{c}\text { Flux }(0.3-2 \mathrm{keV}) \\
{\left[10^{-12} \mathrm{erg} / \mathrm{cm}^{2} / \mathrm{s}\right]}\end{array}$ & $\begin{array}{c}\text { Flux }(2-10 \mathrm{keV}) \\
{\left[10^{-12} \mathrm{erg} / \mathrm{cm}^{2} / \mathrm{s}\right]}\end{array}$ & $\begin{array}{c}\text { photon index } \\
(0.3-10 \mathrm{keV})\end{array}$ & $\chi_{v}^{2}$ & $n_{\text {d.o.f. }}$ \\
\hline July 7 & 55384.044 & $1.0 \pm 0.1$ & $0.9 \pm 0.3$ & $2.0 \pm 0.6$ & 0.2 & 1 \\
July 8 & 55385.048 & $1.9 \pm 0.1$ & $0.6 \pm 0.2$ & $2.5 \pm 0.5$ & 0.8 & 2 \\
July 11 & 55388.803 & $1.8 \pm 0.1$ & $0.6 \pm 0.2$ & $2.5 \pm 0.6$ & 1.1 & 3 \\
July 16 & 55393.031 & $7.1 \pm 0.1$ & $1.2 \pm 0.2$ & $2.9 \pm 0.2$ & 1.1 & 13 \\
July 20 & 55397.047 & $4.3 \pm 0.1$ & $0.9 \pm 0.2$ & $2.8 \pm 0.3$ & 1.0 & 7 \\
July 29 & 55406.818 & $15.2 \pm 0.1$ & $5.8 \pm 0.4$ & $2.5 \pm 0.1$ & 0.7 & 36 \\
Aug. 5 & 55413.024 & $7.3 \pm 0.1$ & $1.2 \pm 0.3$ & $3.0 \pm 0.4$ & 1.0 & 7 \\
Aug. 10 & 55418.049 & $1.8 \pm 0.1$ & $0.8 \pm 0.2$ & $2.3 \pm 0.4$ & 1.3 & 3 \\
Aug. 22 & 55430.218 & $1.3 \pm 0.1$ & $0.6 \pm 0.3$ & $2.3 \pm 0.7$ & 0.4 & 1 \\
Sep. 1 & 55440.594 & $4.9 \pm 0.1$ & $0.7 \pm 0.2$ & $3.0 \pm 0.4$ & 0.4 & 4 \\
Sep. 8 & 55447.090 & $1.7 \pm 0.1$ & $0.7 \pm 0.2$ & $2.4 \pm 0.4$ & 0.7 & 3 \\
Sep. 12 & 55451.852 & $3.5 \pm 0.1$ & $1.7 \pm 0.2$ & $2.3 \pm 0.2$ & 1.5 & 10 \\
Sep. 18 & 55457.194 & $4.5 \pm 0.1$ & $1.5 \pm 0.3$ & $2.5 \pm 0.3$ & 1.3 & 8 \\
\hline
\end{tabular}

Notes. The table includes observation start time (MJD); integral flux in the energy range between 0.3 and $2 \mathrm{keV}$; integral flux in the energy range between 2 and $10 \mathrm{keV}$; power-law photon index, and reduced chi-square $\chi_{v}^{2}$ with the number of degree of freedom $n_{\text {d.o.f. }}$.

Table A.2. Results of Swift-UVOT observation during MWL campaign 2010.

\begin{tabular}{lcccc}
\hline \hline Observation date & $\begin{array}{c}\text { MJD } \\
\text { days] }\end{array}$ & $\begin{array}{c}\text { UV W1 flux } \\
{\left[10^{-11} \mathrm{erg} / \mathrm{cm}^{2} / \mathrm{s}\right]}\end{array}$ & $\begin{array}{c}\text { UV } M 2 \text { flux } \\
{\left[10^{-11} \mathrm{erg} / \mathrm{cm}^{2} / \mathrm{s}\right]}\end{array}$ & $\begin{array}{c}\text { UV W2 flux } \\
{\left[10^{-11} \mathrm{erg} / \mathrm{cm}^{2} / \mathrm{s}\right]}\end{array}$ \\
\hline July 5 & 55382.995 & $1.8 \pm 0.2$ & $3.8 \pm 0.5$ & $2.7 \pm 0.2$ \\
July 7 & 55384.046 & $1.5 \pm 0.1$ & $3.1 \pm 0.3$ & $1.9 \pm 0.2$ \\
July 8 & 55385.049 & $1.4 \pm 0.1$ & $2.9 \pm 0.3$ & $2.3 \pm 0.2$ \\
July 11 & 55388.808 & $1.2 \pm 0.1$ & $2.7 \pm 0.2$ & $1.9 \pm 0.1$ \\
July 16 & 55393.035 & $1.8 \pm 0.2$ & $2.6 \pm 0.3$ & $2.5 \pm 0.2$ \\
July 20 & 55397.051 & $1.6 \pm 0.1$ & $3.8 \pm 0.3$ & $2.8 \pm 0.2$ \\
July 29 & 55406.842 & $2.5 \pm 0.1$ & $5.2 \pm 0.3$ & $4.6 \pm 0.2$ \\
Aug. 5 & 55413.026 & $2.5 \pm 0.1$ & $5.0 \pm 0.4$ & $3.8 \pm 0.2$ \\
Aug. 10 & 55418.075 & $1.8 \pm 0.1$ & $3.4 \pm 0.3$ & $3.0 \pm 0.2$ \\
Aug. 16 & 55424.063 & $2.1 \pm 0.3$ & $3.7 \pm 0.6$ & $2.1 \pm 0.3$ \\
Aug. 22 & 55430.223 & $1.3 \pm 0.1$ & $2.6 \pm 0.2$ & $2.1 \pm 0.1$ \\
Sep. 1 & 55440.595 & $2.5 \pm 0.1$ & $4.6 \pm 0.3$ & $4.0 \pm 0.2$ \\
Sep. 8 & 55447.091 & $1.6 \pm 0.1$ & $2.9 \pm 0.2$ & $2.3 \pm 0.2$ \\
Sep. 12 & 55451.851 & $1.7 \pm 0.1$ & $3.0 \pm 0.2$ & $2.5 \pm 0.2$ \\
Sep. 18 & 55457.197 & $2.1 \pm 0.1$ & $4.3 \pm 0.3$ & $3.4 \pm 0.2$ \\
\hline
\end{tabular}

Notes. The table includes observation start time (MJD), and fluxes for the different three UV filters. 